\title{
Application of Ultrafast Laser Optoperforation for Plant Pollen Walls and Endothelial Cell Membranes
}

\author{
Sae Chae Jeoung, Mehra Singh Sidhu, Ji Sang Yahng, \\ Hyun Joo Shin and GuYoun Baik \\ Korea Research Institute of Standards and Science
}

Korea

\section{Introduction}

Laser beam, first introduced in the field of ophthalmology in 1964 has been a powerful tool in medicine, plastic or general surgery, dermatology, gynaecology, oncology, in vitro fertilization and numerous other clinical areas (Nelson \& Berm, 1989; Senz \& Miiller, 1989). Furthermore, it is also a useful tool for studying basic cellular and biochemical processes. Fluence, energy per unit area, is one of the most important and critical aspects of the laser treatment (Sidhu et al., 2009). Depending upon the laser fluence, either transient or permanent changes can be induced in various cellular compartments including cell walls, plasma membranes, and even organelles with high resolution under physiological environment. For instance, Higashiyama et al. performed a selective ablation of a target compartment of the embryo sac with ultraviolet (UV) pulsed laser to investigate the contribution of each gametophytic cell to pollen tube attraction and found that the synergic cells adjacent to the egg cell plays an important role in attracting the pollen tube (Higashiyama et al., 2001).

Laser pulses with a sufficient spatial resolution can introduce a physical gate in either a membrane or a cell wall under physiological environment such that one could deliver a foreign material into the cell and bring about selective manipulation of subcellular components without demolishing the cell's integrity. It has been reported that in Japonica rice (Oryza sativa), which was pre-treated in a hypertonic buffer containing an exogenous gusA/npt II chimeric gene, however only five out of 1000 treated cells exhibits the gusA gene expression in embryogenic calli (Hoffmann, 1996). In case of tobacco leaves, it has been recently reported that the transferred gene into the tobacco cells via nanosecond laser pulse was stably maintained even during the meiosis. However, the previous reports from nanosecond laser pulses treatment in UV region illustrated a rather limited transformation efficiency of less than $0.5 \%$. The shortage in transformation efficiency can be overcome by utilizing opto-acoustic shock pressure induced by fundamental output from Nd:YAG pulsed laser (Tang et al., 2006). Since pollen grain is a haploid and is known to be regenerated into a plant, this laser application draws a great interest from the application point of view in plant transformation and genetic engineering. Nevertheless, the cell wall of plant cell including pollen is not easily accessible because it forms a potential barrier for the

Source: Advances in Lasers and Electro Optics, Book edited by: Nelson Costa and Adolfo Cartaxo, ISBN 978-953-307-088-9, pp. 838, April 2010, INTECH, Croatia, downloaded from SCIYO.COM 
active or passive transport for the molecules. This problem can be resolved via utilizing the cell protoplast as a carrier for the molecules, but this step may add the numerous other problems such as, tissue disintegration and difficulties in plant regeneration (Krautwig \& Lörz, 1995). More recently, ultrafast laser-based gene transfer has been considered as a break-through in the current hiatus of non-viral mediated gene therapy (Greulich \& Weber, 1992). Compared to relative intensive work on mammalian cells, application of femtosecond-laser optoperforation in plant cells is quite limited. However, the application of the recently found ultrafast laser ablation technique in the plant cells is expected to bring about a significant increase in gene transfection efficiency by eliminating physical barriers such as the hard cell wall or the turgor pressure (Tirlapur \& König, 2002).

It is important to understand the developmental process of a pollen including germination of a receptive stigma and consequent tube growth as a basic sexual mechanism in flowering plants for the manipulation of plant production (Taylor \& Hepler, 1997). Pollen transformation is also considered as an alternative strategy for the genetic engineering. Pollen acts as a natural vector for direct gene transfer due to its involvement in the normal sexual process in flowering plants and hence, transformed pollen has been used to generate numerous transgenic plants such as Tobacco, Scots pine etc., (Van der Leede-Plegt et al., 1995; Touraev et al., 1997; Aronen et al., 2003). In addition to several other potential advantages of this technology like rapid propagation with reduced somaclonal variation, pollen transformation does not require any in vitro regeneration process that is typically essential for genetic engineering. For instance, for the restoration of American chestnut which is difficult to regenerate, in vitro, pollen transformation based on particle bombardment method was successfully applied to transiently express green fluorescence protein (GFP) with an efficiency of $4.1 \%$ (Fernado et al., 2006).

In first section of this chapter, we are discussing the application of ultrafast laser optoperforation to make a transient hole on lily (Lilium hybrid cv Shiberia) pollen wall to deliver the desired foreign material into the pollen, which furthermore improve its transfection efficiency and also eliminates the tedious in vitro regeneration or tissue disintegration problems. The presence of apparent holes on the cell wall, after single-shot laser irradiation, was confirmed by measuring the AFM topography of the processed pollen surface. Laser exposer with a moderate to high laser fluence induces a massive extrusion of the pollen's interior contents through the hole. However, the in vitro germinability of perforated pollens was comparable to those of the untreated ones. If one of the two nuclei compartments of the pollen grains, which are tentatively assigned to be a generative cell, was excluded out from the pollen wall through the transient hole, the second one (vegetative cell) moved along the tube tip endorsed to follow the normal pollen tube growth dynamics. This reveals that during the germination, coherent movement of both the nuclei is not a necessary requisite for normal pollen tube growth. Meanwhile, we have tried and successfully able to deliver a foreign materials into the pollen grain, which results in a transient red fluorescence protein (RFP) expression with a high efficiency. The use of such an optoperforation followed by the delivery of foreign material into the pollens holds an imperative approach in the field of pollen germination, growth of the pollen tube and the pollen transformation.

We will devote the latter part of this chapter to explain the ultrafast laser-assisted optoperforation of endothelial cell membrane. In order to induce the transient perturbation on the live-cell plasma membrane for the efficient delivery of foreign materials, including drugs or genetic material, into the live cells (such as embryonic stem cell), numerous 
chemical or physical methods have been employed (Kobayashi et al., 2005). Also, it's an ever chasing challenge waiting for us to elucidate the mechanism involved in the self-healing process of the perforated live cell plasma membranes. The mechanism for laser-mediated foreign material transfer as well as other perturbation methods was hypothesized with a limited understanding of the structure and functions of membrane lipids (Engelman, 2005). Recently, the fundamental mechanisms of fs-laser interaction with an aqueous media are extensively studied by Vogel et al. (Vogel et al., 2005). The most frequently invoked explanation was based on the assumption that the physicochemical methods used in the specific cases may either perforate the surface of the cell membranes or enhance the permeability of the foreign materials, enabling a transient transfer of foreign molecules into the cytosol (Kobayashi et al., 2005; Zeira et al., 2003). Manipulation and operation using ultrafast laser pulses have been introduced as a promising tool for the cellular nanosurgery and the transfer of foreign molecules into live cells under in vitro and in vivo conditions (Zeira et al., 2003; Tirlapur \& König, 2002; Shen et al., 2005; König et al., 1999). Ultrafast laser-based gene transfer has been considered as a break-through in the current hiatus of non-viral-mediated gene therapy (Zeira et al., 2003). Lastly, in this chapter we are discussing the experimental results on the optoperforation of retina blood vessels with minimizing any probable damage outside laser focusing area.

\section{Ultrafast laser optoperforation of lily (Lilium hybrid cv Shiberia) pollen}

\subsection{Materials and method}

Mature pollens were obtained from the anthers of Lilium hybrid cv Shiberia. After collection, the pollens were air- dried and stored at 4o C. Before in vitro culture, pollens were rehydrated in deionized water under humid atmosphere for about $30 \mathrm{~min}$ and spread on the culture medium ( $\mathrm{pH} 5.8$ ) comprising of $145 \mathrm{mM}$ sucrose, $1.6 \mathrm{mM} \mathrm{H}_{3} \mathrm{BO}_{3}, 1.8 \mathrm{mM} \mathrm{Ca}\left(\mathrm{NO}_{3}\right)_{2}$ and $0.4 \%$ phytagel (Sigma).

Near-infrared laser beam from amplified Ti:sapphire femtosecond laser at a repetition rate of $1 \mathrm{kHz}$ (Quantronix) was focused on the pollen exine through objective lens (N.A. $=0.6$ ) so that we could selectively puncture a wall inside an ornament. Petri dish containing the pollen grains was mounted on a motorized XYZ - translation stage, which is used to manipulate the sample to expose a fresh pollen grain surface at each laser pulse. The images obtained during the fs-laser irradiation on the pollen's surface were fed into a personal computer for further data analysis. Laser fluence was attenuated by a variable neutral density filter. All the laser exposers were conducted under a single shot configuration by using a fast mechanical shutter with a opening time constant of $0.6 \mathrm{~ms}$, which is synchronized with the laser. After optoperforation, the viability of the operated pollen grains was checked by measuring the germination rate of pollen tube by intrusion of propidium iodide (PI). The glass coverslips with grids marked on the surface, were used to cover over the pollens for the localization of the irradiated pollen grains.

For the pollen transformation study, a foreign DNA with a construct, encoding F1TPae- $\gamma$ mitochondrial targeting sequence fused to RFP; was added into the culture medium before the laser optoperforation (Lee et al., 2001). MitoTracker Green FM (Molecular probes, invitrogen) was further used to confirm the RFP expression. MitoTracker Green FM is a conventional fluorescent marker used for tracking of the mitochondria (Lovy-Wheeler et al., 2007).

Pollen germination as well as its response to optoperforation was characterized with an optical microscope and a fluorescent microscope (Axioskop, Zeiss). In order to confirm the 
hole size, the intact pollen surface treated with laser beam was imaged by atomic force microscope (XE-120, PSIA) without any further treatments.

\subsection{Results and discussion}

It was observed that size of lily pollen grain increased during pollen re-hydration process. Before pollen tube starts to grow, a laser beam was precisely focused into the interior of the ornament of pollen surface. Figure 1 shows sequential optical microscopic images captured from the movie file obtained during fs-laser optoperforation of lily pollen walls. Immediately, after laser pulse irradiation on pollen wall surface, a cytoplasmic content of the pollen oozes out from the pollen grains.

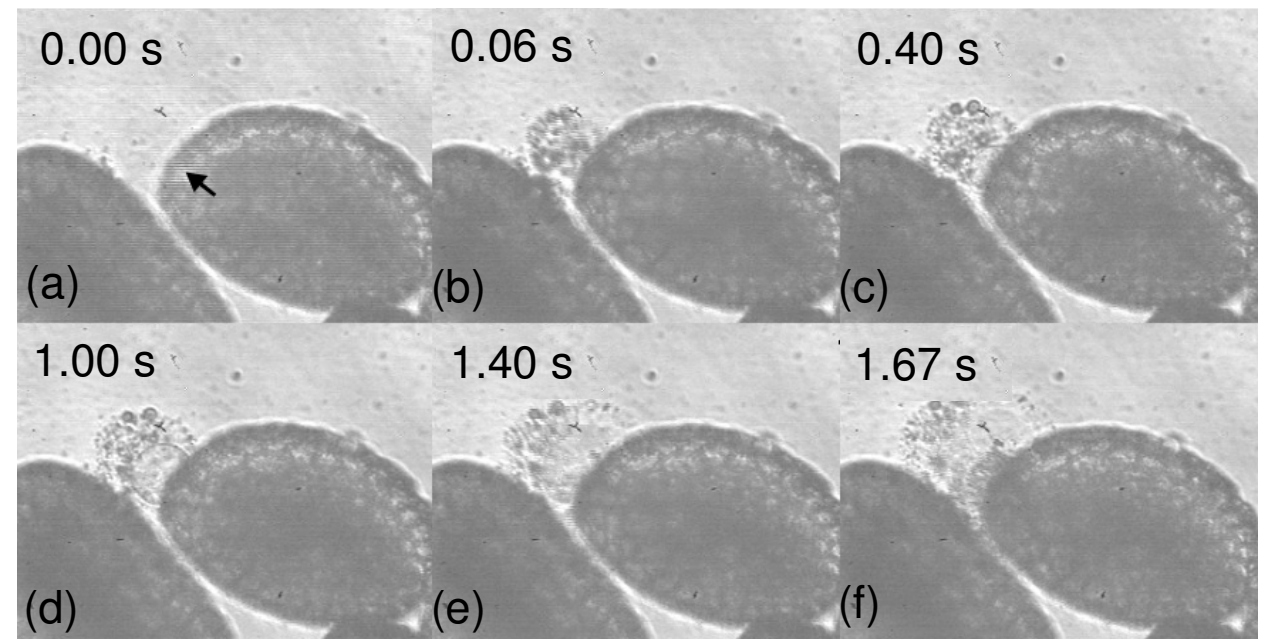

Fig. 1. Sequential images of hydrated lily pollens before (a) and after fs-laser treatment (b-f). The contents of pollens were burst out just after laser irradiation on the selected surface with a time span of 0 to 1.67 seconds. The arrow indicates the point of focusing.

This observation is consistent with the previous reports stating that UV laser pulses treatment causes release of cytoplasmic content either from the pollen itself or its tube (Hoffmann, 1996). This abrupt extrusion of cytoplasm from the treated pollen can be explained in terms of a high turgor pressure inside the hydrated pollen, which ranges between 0.1 and $0.4 \mathrm{MPa}$, and the mean value being $0.21 \pm 0.06 \mathrm{MPa}$. (Benkert et al., 1997). Unless the buffering osmotic pressure is against the turgor pressure, the pressure inside the cell will no longer sustain due to the presence of hole on the wall. Following the cytoplasmic extrusion, a large vesicle with a diameter of about $10 \mu \mathrm{m}$ was also flushed out from the pollen grain through the hole. The excluded large structure was apparently distinct from the concomitant flushed contents, as seen under optical microscope. The distinct boundary was rather rigid in nature and it remains intact without any bursting phenomenon.

The extrusion of pollen's cytoplasmic contents and the large organelle was strongly dependent on laser fluence. Figure 2 shows a series of optical microscopic images and a graph that represents the normalized germination percent of the pollen grains as a function of the laser fluence $\left(0.11-7.10 \mathrm{~J} / \mathrm{cm}^{2}\right)$. With changing the laser fluence energy from 0.11 $\mathrm{J} / \mathrm{cm}^{2}$ to $7.10 \mathrm{~J} / \mathrm{cm}^{2}$, we perforate the pollen walls with single fs-laser pulse. Figure 2(a) 


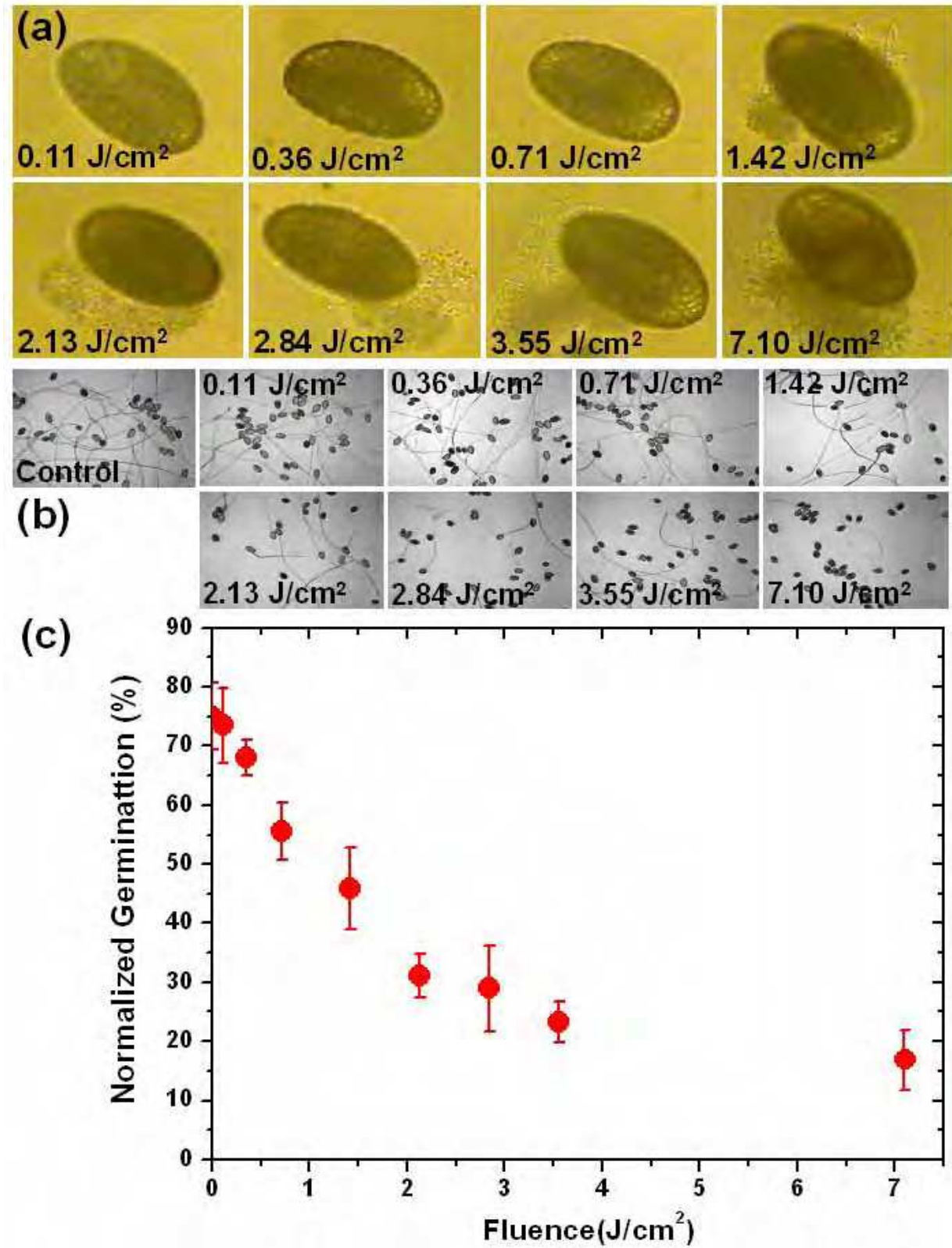

Fig. 2. The percent germination threshold of the hydrated (single pulse laser irradiated) lily pollen grains (a) Optical microscope images (b) Germination of the pollen grains after laser irradiation at varied fluence range $\left(0.11 \sim 7.10 \mathrm{~J} / \mathrm{cm}^{2}\right)$. (c) Graphical representation of Normalized germination percent of the pollen grains as a function of laser fluence. The pollen wall ablation threshold was found to between $0.71 \sim 1.42 \mathrm{~J} / \mathrm{cm}^{2}$. 
shows the exclusion of pollen's cytoplasmic contents at progressive laser fluence energy levels. We observe that the hydrated lily pollens mostly begin to exclude cytoplasmic contents at the laser fluence energy level of $1.42 \mathrm{~J} / \mathrm{cm}^{2}$. Sometimes we can observe the extrusion of the cytoplasmic contents even at the laser fluence less than $1.42 \mathrm{~J} / \mathrm{cm}^{2}$. We have also compared the germinabilty rates of treated $v$ s non-treated pollen grains at above mentioned laser fluence energy levels. With the optical setup used in the current work, the threshold laser energy for making a physical hole on the wall was found to be about 0.5 $\mathrm{J} /$ pulse, where there is no observable extrusions of any cytoplasmic contents were seen out the pollen grains. Figure 3 shows the AFM topography of perforated pollen surface. The diameter and depth of the hole is found to be $1.1 \mu \mathrm{m}$ and $0.9 \mu \mathrm{m}$, respectively.

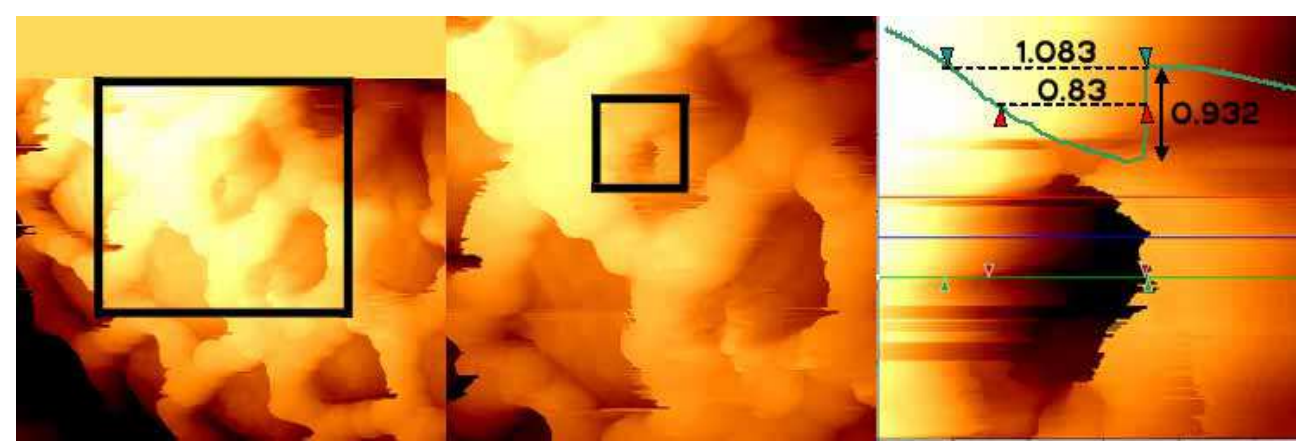

Fig. 3. Inset represents the AFM image of hole after being perforated at $0.5 \mathrm{~mW}$ fs-laser pulse. The extrusion of any cytoplasmic contents out of the pollen grains was not observed at the said fluence.

We have cross-checked the viability of the pollen grains after optoperforation by the observation of intrusion of the PI (Propodium Iodide). About 150 out of 300 opto-perforated pollens were not stained with PI despite of the massive exclusion of the cytoplasmic contents, indicating that the fs-laser optoperforation of lily pollen wall hardly harms its viability. More interestingly, the excluded large organelle was not stained with PI, even though the organelle was not likely surrounded by pollen wall. This observation led us to assume that the organelle excluded from the pollen grains by the fs-laser processing still maintained its biological integrity without any observable perturbation.

Figure 4 exhibit a series of optical microscopic images during the in vitro germination of pollen after optoperforation at a time lapse of 0,4 , and $11 \mathrm{hrs}$. The pollens operated with the fluence of $8 \mu \mathrm{J} /$ pulse are indicated with asterisk. Even though the contents of pollen including the large organelle were massively excluded just after optoperforation of cell wall, still $19 \%$ of the operated pollens (480 pollens in total) were tend to germinate the pollen tubes successfully. This germination efficiency is very close to the value of $24 \%$ as observed under the control experiment (420 pollens in total).

Subsequently, we emphasized our study on nucleus staining of the treated pollens to have a deeper understanding on the nature of excluded large organelles. Figure 5 shows bright-field optical image (a) and fluorescence image (b) of pollens stained with nucleic acid specific dye acridine orange after the fs-laser treatment (1, 2, and 3 labelled pollens). Cellular contents were massively extruded from all of the treated pollens upon illuminating rather at the high laser fluence $(\sim 8 \mu \mathrm{J} /$ pulse). Amongst them, only pollen " 1 " has been successfully 


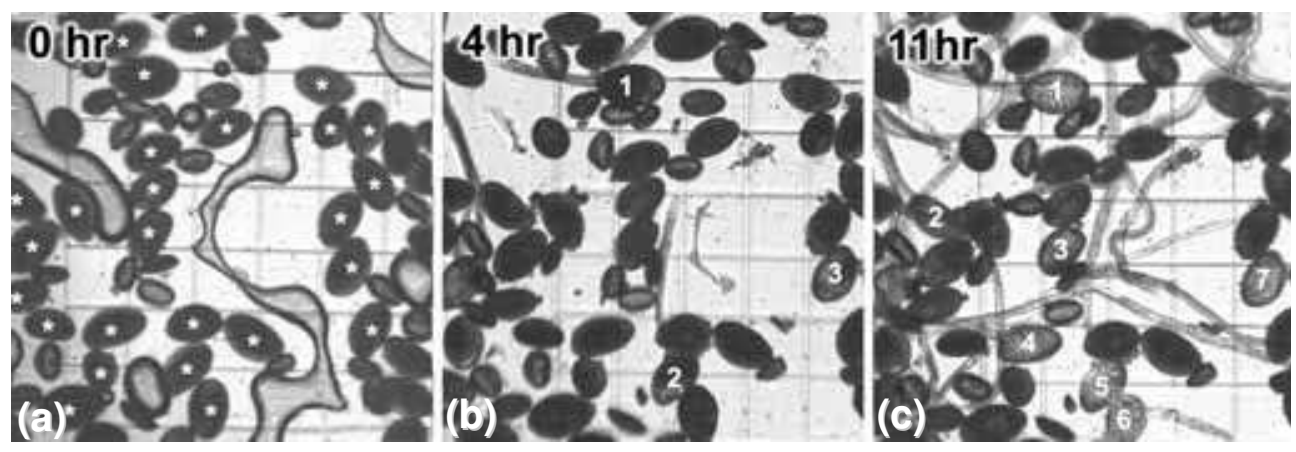

Fig. 4. Sequential images of pollen tube growth before (a) and after fs-laser treatment (b-c). fs-laser beam was focused into the interior of the wall inside pollen ornament. The pollens marked with asterisks were irradiated with laser beam with the fluence of $8 \mu \mathrm{J} /$ pulse. Seven pollens out of 26 treated pollens in this run were eventually geminated to grow the pollen tube.

germinated to grow the pollen tube. It should be noted that an apparent fluorescent spot (arrow), indicating the presence of nucleic acid, was observed in the excluded contents in all the treated pollen grains. A faint emission indicated by an arrow head, which supposed to be a vegetative nucleus, was clearly observed in the pollen tube geminating from pollen " 1 ". Meanwhile, the nuclei excluded from the same pollen were obviously seen outside the pollen grain. Thus, it is most likely to state that the excluded large vesicle from the pollen "1" must either be a generative cell with its own nucleus or a sperm cell after the second mitotic division. In other words, the vegetative nucleus of treated pollen " 1 " is well trapped/tracked inside the tube, while the regenerative nucleus or sperm cell stayed outside the pollen. Vervaeke et al. suggested that the vegetative nucleus (VN) and the generative cell (GC) form a functional assemblage to generate callose plugs (Vervaeke et al., 2005; Strubinska \& Sniezko, 2000). However, our findings clearly indicate that the close physical contact between the vegetative nucleus and the generative cell is not a necessary requisite for the pollen germination. Instead, it suggests a kind of chemical connection between the two constituents. Even if it is not known as how the two constituents are connected physically or chemically, the extrusion of generative cell from lily pollen grains by fs-laser optoperforation does not alter the growth of pollen tube or vegetative nucleus movement to a greater extent.

We have further performed the transformation studies for the fs-laser treated lilium pollen grains. Initially the pollen grains were soaked in hypotonic solution having a foreign DNA with a construct encoding F1TPae- $\gamma$ mitochondrial targeting sequence fused to RFP. An apparent expression of RFP from the optoperforated pollens as shown in fluorescence microscopic images (Figure 6) reveals that the current optoperforation method could be successfully applied to transfer the foreign genetic materials into the lily pollens. Figure 7(a), after 72 hours, represents the successful germination of the pollens, treated with an fs-laser beam. From the fluorescence image of germinated pollens (Figure 7(b)) measured at $650 \mathrm{~nm}$ (wavelength corresponding to the emission from RFP), we deduced that the DNA delivered into the pollen grain was effectively expressed in pollen tube during the germination due to successful transformation. We have further confirmed transformation of the lily pollen by staining the transformed pollen tube with MitoTrack Green FM, which selectively stains the 


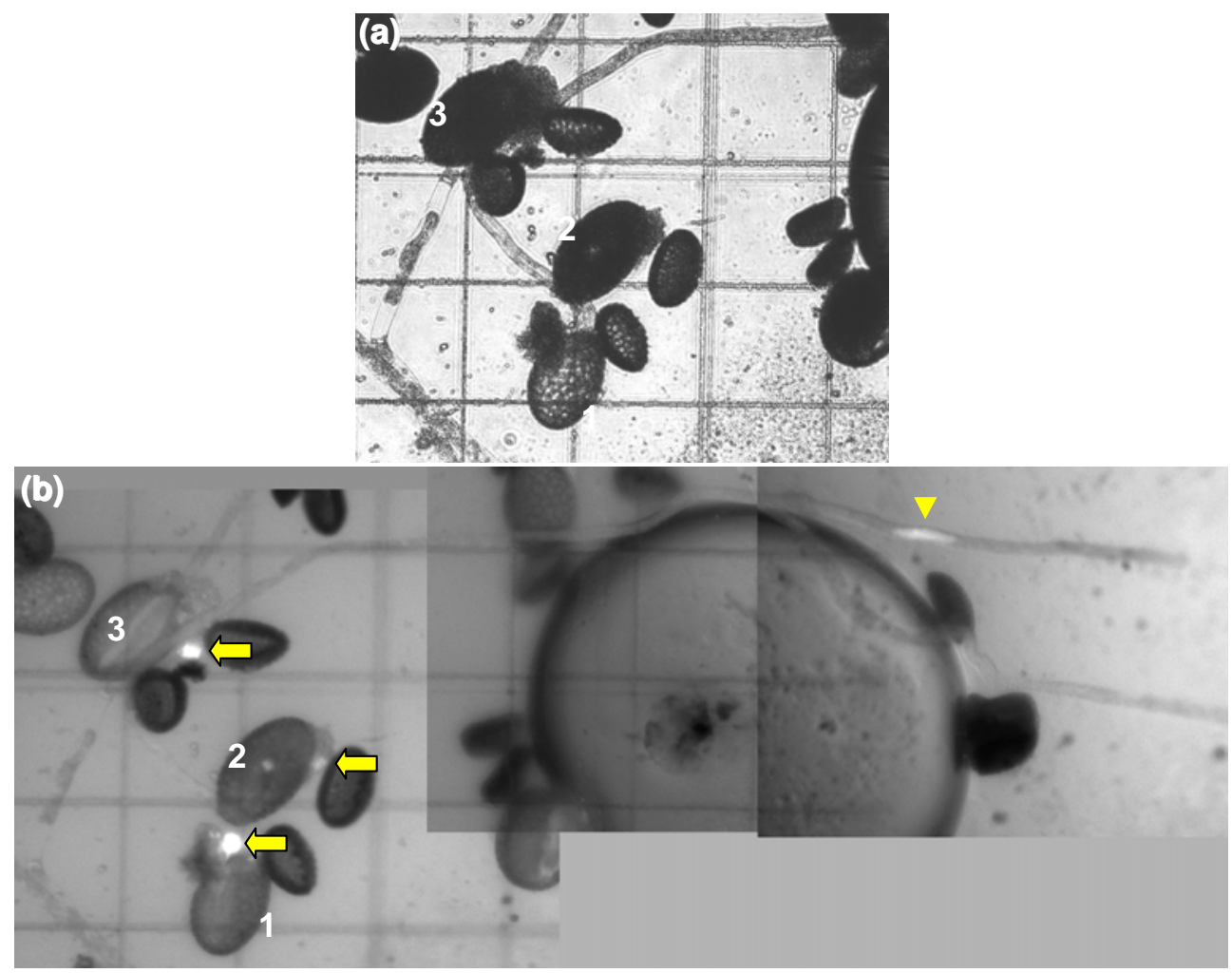

Fig. 5. Bright field (a) and fluorescence label (b) of germinated pollens ("1", “2", and "3") stained with nucleic specific dye, acridine orange after fs-laser treatment. Interior cytoplasmic contents were massively excluded from all the treated pollens due to rather high laser fluence. Amongst them, only pollen "1" successfully germinated to grow the pollen tube. It should be noted that an apparent fluorescent spot (arrow) due to the presence of nucleic acid was observed from the excluded contents from all the treated pollen grains. A dim emission part (arrow head), which should be vegetative nucleus, was observed in the pollen tube geminating from treated pollen " 1 ". The nucleus excluded from the same pollen was very well seen.

mitochondria and results in the expression of green fluorescence. Exact matching of RFP fluorescence image with that of mitochondria in pollen tube led us to conclude that, the foreign DNA with a construct encoding F1TPae- $\gamma$ mitochondrial targeting sequence fused RFP was delivered into pollen and expressed as designed. Statistically, 42 pollens out of 206 treated pollens were germinated. Amongst the germinated pollens, foreign DNA expression in mitochondria delivered by fs-laser optoperforation was observed in 9 pollen tubes out of 42 germinated pollens. As a result, the transformation efficiency of lily pollen by fs-laser optoperforation was about $4.4 \%$. However, in case of germinated pollens, the efficiency was about $21.4 \%$. This transformation efficiency was higher than that of American chestnut pollen transformation based on particle bombardment method to transiently express GFP with an efficiency of $4.1 \%$ (Fernado et al., 2006). 


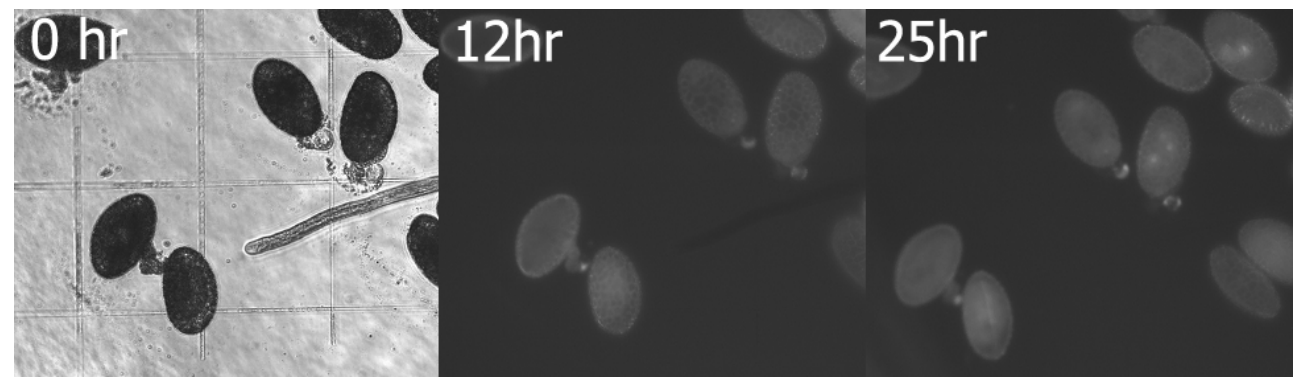

Fig. 6. The red fluorescence images were taken at 0,12 and $25 \mathrm{hr}$ periodic intervals of time. The fluorescence signal at the membrane site was thought to be due to expression of foreign DNA.

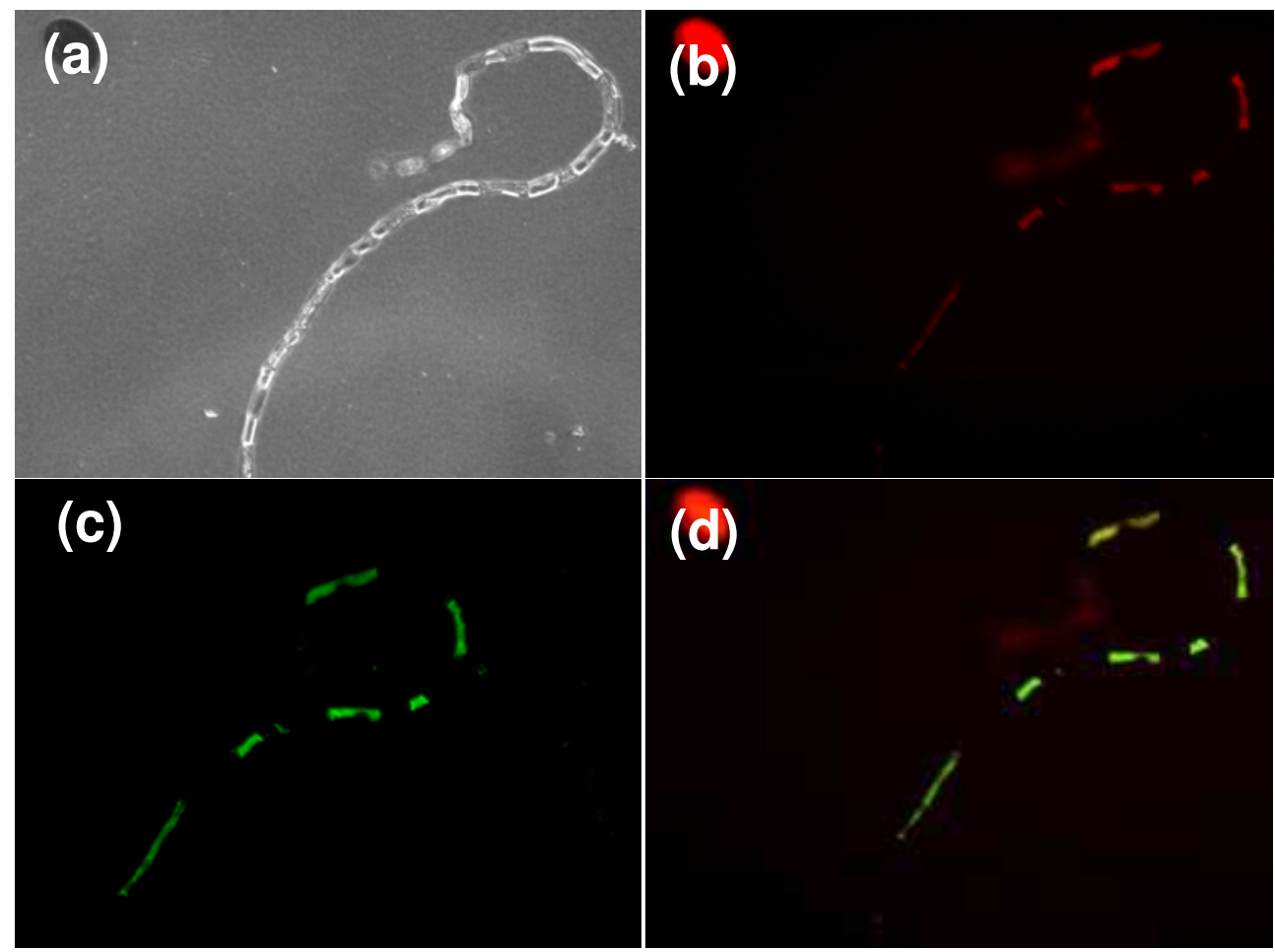

Fig. 7. Direct proof for the selective transformation of a foreign DNA with a construct encoding F1TPae- $\gamma$ mitochondrial targeting sequence fused to RFP in the mitochondria of a pollen tube. (a) DIC microscopy, (b) RFP fluorescence, (c) MitoTracker green fluorescence and $(d)$ merge $b$ and $c$ 


\section{Ultrafast laser optoperforation of endothelial cell membranes}

\subsection{Experimental techniques and materials}

The perforation of the plasma membrane of live cells was performed with a femtosecond amplifier system (Integra, Quantronix) with maximum output energy of $1 \mathrm{~mJ} /$ pulse at 780 $\mathrm{nm}$, of which the pulse width and the repetition rate is $150 \mathrm{fs}$ and $1 \mathrm{kHz}$, respectively (Jeoung et al., 2005). The laser beam was tightly focused on the membrane with a highnumerical-aperture objective lens (Nikon, X100, N.A $=1.3$, oil immersion lens) mounted to an inverted microscope (Axiovert 200M, Zeiss). All the experiments were done under singleshot configuration by using a fast electrical shutter with an opening time constant less than $0.5 \mathrm{~ms}$. In order to eliminate any desperate loss of laser energy during these experiments, the fast electrical shutter was perfectly synchronized with the laser. The laser energy before the objective lens was kept to be about $5 \sim 7 \mathrm{~nJ} /$ pulse by attenuating it with a neutral density filter.

The changes in the topography and the optical images of plasma membrane induced by the ultrafast laser irradiations were simultaneously monitored with both an atomic force microscope (AFM) and a charge coupled device (CCD) detector, respectively. AFM (XE-120, PSIA) was slightly modified and combined to an optical microscope. AFM probe was carefully aligned to be aimed at same position of the focus of fs-laser beam. Contact imaging of the AFM was performed using a silicon nitride bio-cantilever (Bio-lever, Olympus, Japan), of which nominal force constant is $0.03 \mathrm{nN} / \mathrm{m}$. The topographies have been obtained by optimising the AFM running parameters that gives the identical results to that of the two-way scanning configuration (Parpura et al., 1993 \& Velegol et al., 2003). The set point for loading force was kept to be very weak between $0.65 \mathrm{nN}$ and $1 \mathrm{nN}$, to minimize the damage or the attractional movement caused by attraction force of the AFM tip. A successive scanning of the live cells exhibit no observable changes in its phenotypes.

The measurements of the velocity field responsible for the membrane motility after fs-laser perforation have been measured by a micro particle image velocimetry ( $\mu$-PIV) (Yahng et al., 2005). The visualization of membrane movements was figured out by attaching a fluorescent polystyrene bead (diameter $\sim 200 \mathrm{~nm}$ ) on the plasma membrane of a target cell. Just after the fs-laser irradiation on the surface of plasma membrane, two successive ns-laser pulses with a time lapse of $10 \mathrm{~ms}$ were employed to capture the two fluorescence images. Thereafter, the velocity fields of membrane motility were obtained by a cross correlation calculation with the two images. We have confirmed that there is no damage on the cells with the current volumetric excitation with the ns-laser pulses, where the laser fluence is kept explicitly low. Neuroblastoma (Neuro2A) cells were used to investigate the dynamics of its plasma membrane and self-healing process. During the experiment, live cells were kept inside a sterile miniaturized cell chamber filled with Dulbecco's Modified Eagle's Media (DMEM, Gibco BRL) containing $10 \%$ Fetal Bovine Serum (FBS, Gibco) and $1 \%$ penicillin/streptomycin (Gibco) under humid atmosphere at $5 \% \mathrm{CO}_{2}$ partial pressure. Although the lid glass of the cell chamber was removed for AFM imaging or other measurements, no apparent change in its phenotypes was observed within time span of (1.5 hr) the current experiments.

\subsection{Results}

As a consequence of single-pulse ultrafast laser irradiation on the cells surface (ca. $5 \sim 7 \mathrm{~nJ}$ ), a circular area appears to be observed on its plasma membrane as shown in Fig. 8(a). The 
injured area increased relatively fast over several tens of seconds comparable to the percentage of cellular size. It is interesting to note that the initial circular type of injured membrane area changes to an elliptical one with a long axis parallel to the cell. After
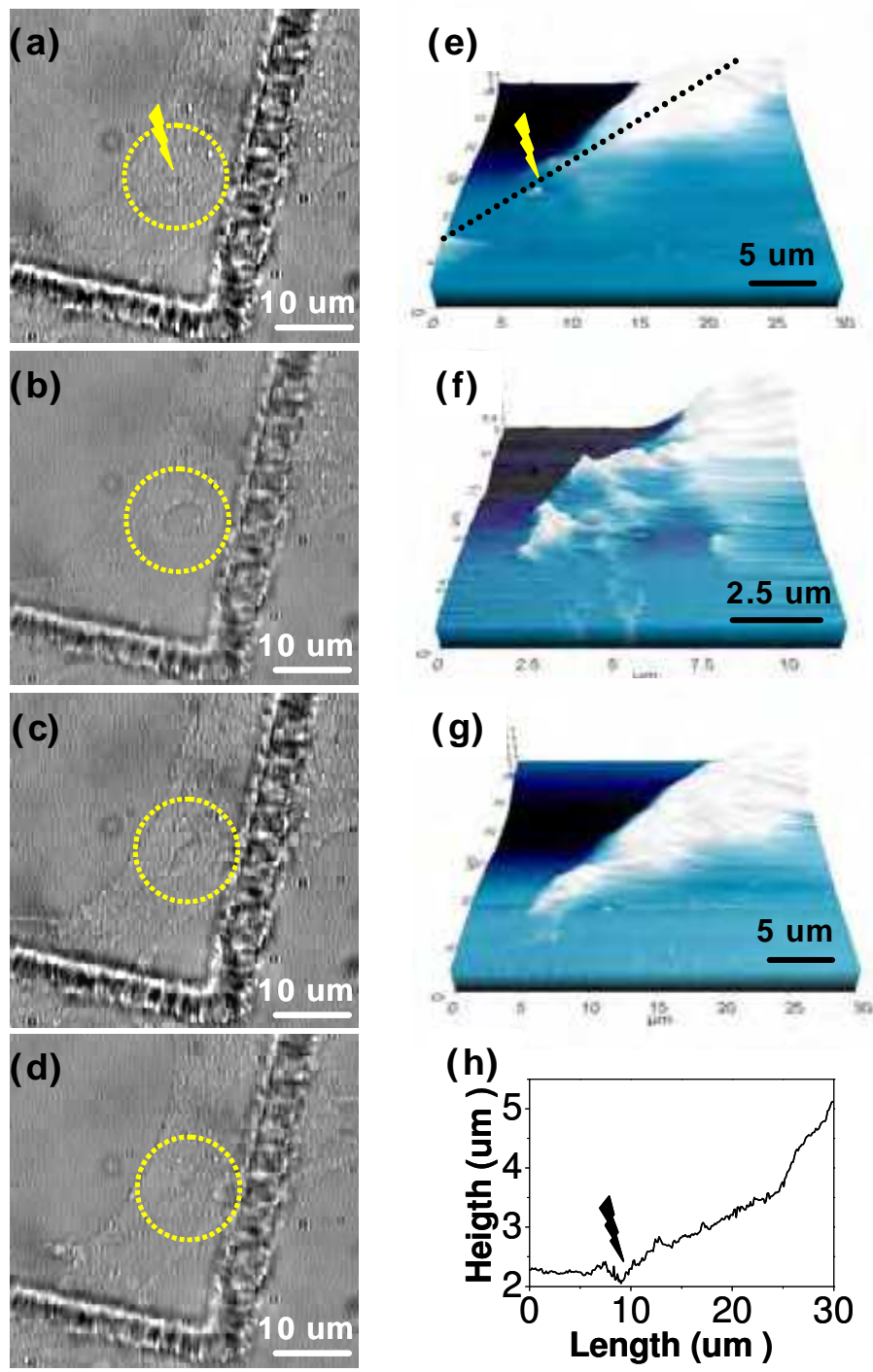

Fig. 8. Transparent photomicrographs (a-d), which were taken $0 \mathrm{sec}, 38 \mathrm{sec}, 229 \mathrm{sec}$, and 414 sec, respectively, and topographical images (e-g) taken 1, 30 and 43 minutes, respectively, after ultrafast laser-induced injury in a single Neouro2A cell. The height profile along the broken line in (e) is shown in (h). The image sizes in (a-d) were $50 \mu \mathrm{m} \times 50 \mu \mathrm{m}$. The hollowed black area is a remnant of fs-laser ablated region, which was processed on the substrate as a guideline before seeding of the cells. 
reaching at its maxima of about $6.4 \times 4.9 \mu \mathrm{m}^{2}$, within several tens of seconds (Fig. 8(b)), the area starts decreasing spontaneously and then completely faded out in next 7 minutes (Fig. $8(\mathrm{c}-\mathrm{d})$ ). We have checked the cell viability using trypan blue-dye extrusion and found no evident change in laser treated cells compared to that of intact cells. AFM topography shown in Fig. 8(e-g) were taken with a time-laps after irradiation. Fig. 8(h) exhibit a typical cross-sectional height profiles along dotted line in Fig. 8(e). It shows the presence of a deep hole on the irradiated plasma membrane, of which diameter is about $1.6 \mu \mathrm{m}$ at a full width at maximum. Meanwhile, the estimated initial size must be the upper limit due to the limitation of spatial resolution for optical detection as well as the limitation in scanning speed for topographical imaging by the AFM. However, the laser beam irradiations on the fixed cells under the similar experimental conditions leads to a submicron-sized selective ablation (for dissection) and hole drilling on the plasma membrane surface. With a repeated scanning of the injured membrane, the topographical image shows not only the spontaneous movement of the ruptured region on the plasma membrane but also the dynamic changes in its shape as well as its size.

While the presence of injured area was completely faded out in the optical images within a time span of several minutes after the perforation, further changes on the membrane surface were still observed in AFM topographical images as shown in Fig. 8 (f). The observations from the topographical images reveals that the highly localized irregular ruffled features along with small irregular movements after the laser irradiations are in-part an experimental observations for the self-healing process of the plasma membrane (Fig. 8 (f)). After about 1 hour, the plasma membrane was found to be fully recovered in its topographic feature and looks almost similar as that before the perforation. Accompanied with these dynamic changes in plasma membrane, a trace tailed to the operated cellular parts, which is initially absent as shown in Fig. 8 (e), appeared on the substrate surface after several minutes of the perforation. Close examination of the shape and position of the trace for laser perforations indicates a little cellular or cytosolic extrusion along with the progressive increment in holesize.

Figure 9 exhibits the temporal behavior of the geometrically averaged radius of the hole induced by fs laser ablation. The temporal behavior of the injured area was found to be strongly dependent on the pulse energy and the irradiated position.

For all the cases, where the laser energy is in between $5 \mathrm{~nJ}$ and $7 \mathrm{~nJ}$, a very small quantity of the cellular contents are extruded from the injured cells before complete disappearance of the changes at site of injured area. With the progressive rise in the laser fluence, initially there is a successive increase in the injured area verified by the increments in radius of perturbation. However, at the higher fluence levels, the cell contents are massively extruded from the cells results in the complete rupturing of the injured cells without any initiation of self healing process. Upon irradiating the cells towards the site of nucleus, the laser fluence even lower than $5 \mathrm{~nJ}$ results in complete rupturing of the plasma membrane. These observations suggest that geometrical factors of the plasma membrane of the live cells plays an important role in the dynamics of ruptured membrane of live cells, assuming that the physicochemical nature of the plasma membrane is isotropic across the entire live cell.

We have an access to the velocity field by attaching the fluorescent beads (diameter $\sim 200$ $\mathrm{nm}$ ) on the surface of the plasma membrane with several time lapses (Fig. 10). After incubation of the cells with the beads for about 12 hours, a strong correlation between fluorescence and topographical image from the beads, led us to know that the beads were 


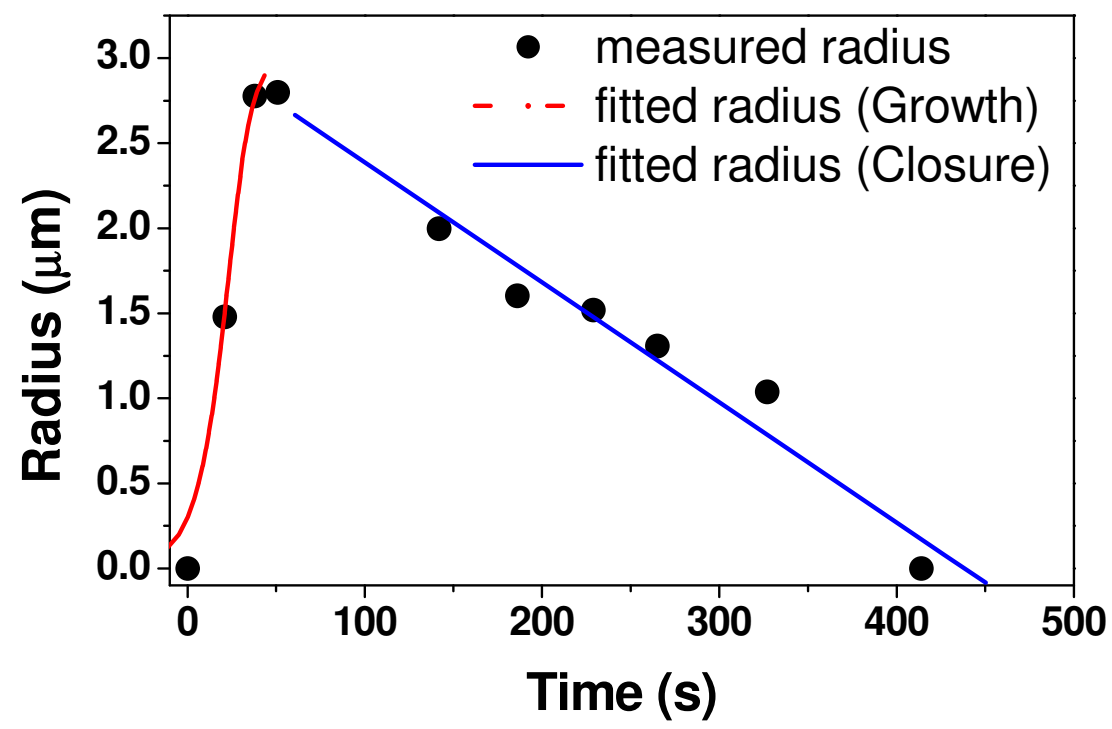

Fig. 9. Temporal behavior of the geometrically averaged radius of hole on the plasma membrane perforated by fs laser. The growth of the holes was fitted with the proposed model using Eq. (3) with the parameters of $r_{i}, r_{c}$, and time constant $t$ of $0.3 \mu \mathrm{m}, 3 \mu \mathrm{m}$ and 12 $\mathrm{sec}$, respectively. For the closure of the hole, the least-square fitting procedure on the experimental data gives the speed of about $0.007 \mu \mathrm{m} / \mathrm{sec}$.

not delivered into the cytosol but remain attached on the surface of plasma membranes. With an aid of applied force to the beads by AFM tip, we found that interaction force between the beads and plasma membrane is fairly high enough to visualize the membrane dynamics without any detachment of the beads during the dynamic membrane movements. Just after the laser irradiation, the velocity field is found to be radial in approximately with a direction towards the edge of the cells. The magnitude of the velocity field decreased with increasing the distance from the position of irradiation. This suggests that all the lipids collected from the growing hole are almost homogeneously spread throughout the remaining area of plasma membrane. Furthermore, the absence of any rim collecting the lipids associated with a long-range flow of the membrane indicates a viscoelastic behavior of the membrane opening process. The velocity field of the membrane closer to the initial ruptured area turns to the opposite direction in next 0.7 seconds after the perforation, while the plasma membrane at a long distance from the irradiated area is keeps on expanding. Afterwards, the movement of velocity fields for the membrane looks randomized, as shown in Fig. 10(d) and (e). Followed to these interesting dynamical behaviors of the live cell membrane, overall pattern of the velocity field in both direction and magnitude resumed again, as observed just after the perforations previously (Fig. 10(f)). At a short span of time, the repeated change in direction of velocity field accompanied with an ultimate hole-size increment seems to be due to an elastic propagation. It behaves like a rubber, with fairly a high elastic modulus. After a long overall hole opening time, the velocity fields of membrane movement turned its direction towards the initially injured area, possibly be associated with the self-healing process of the created holes. 

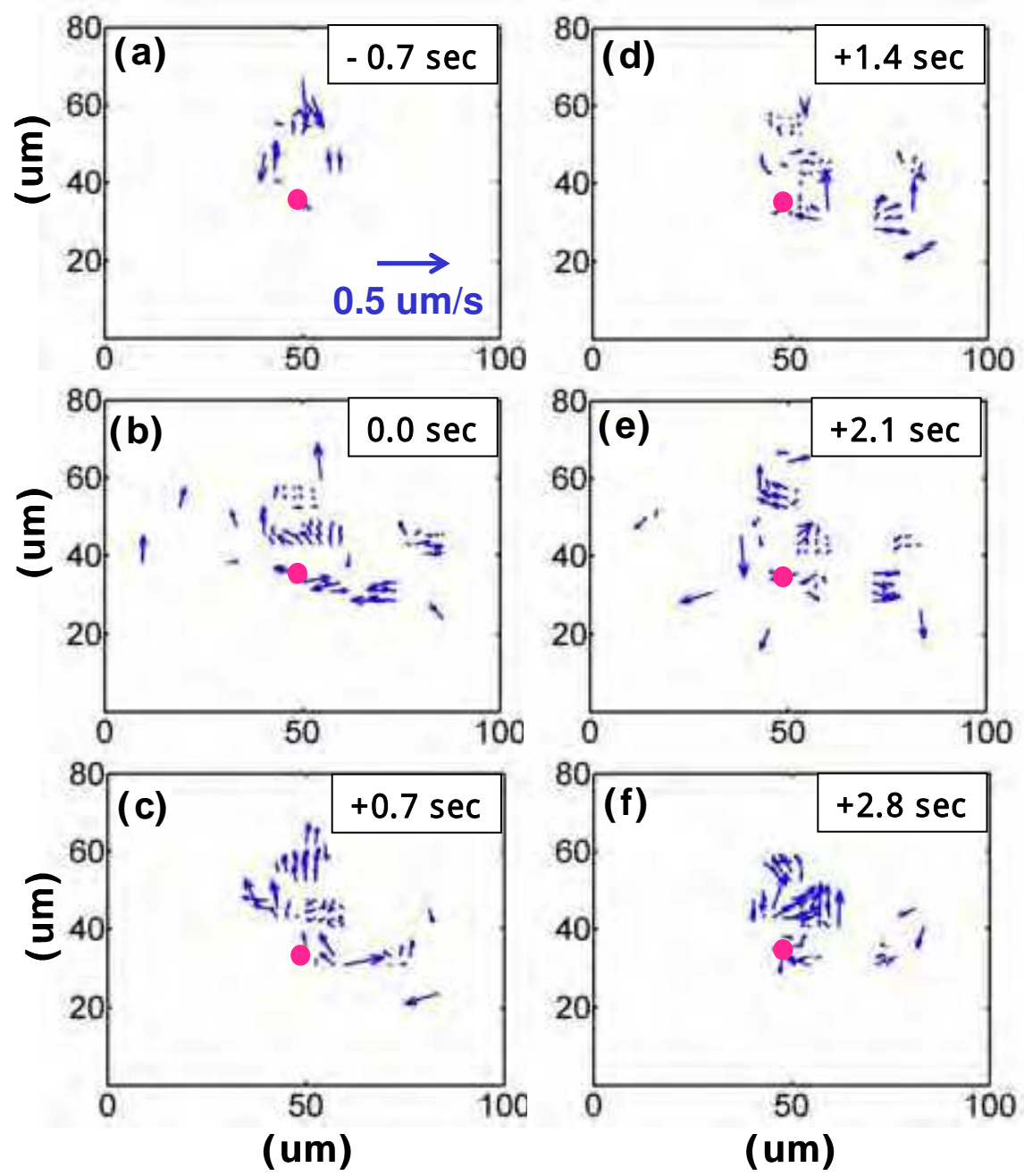

Fig. 10. Time sequence of the measured velocity components and contours of the plasma membrane associated with the hole opening of the plasma membrane of live N2A cells. The red circle indicates the point of laser irradiation.

\subsection{Discussion}

The simplest explanation for the mechanism of laser-mediated foreign material transfer is based on the assumption that the foreign material added to the transforming medium passively enters into the cell through the laser-induced membrane perforation. Furthermore, it successfully undergone with a rapid self-sealing process follows immediately with no subsequent and irreversible injury to the cell (Zeira et al., 2003). The results demonstrated in the current study made the above assumptions true for the Neuro 2A cells, where the cells were irradiated with an ultrafast laser in near infrared (NIR) region. 
The dynamic behavior of the rapidly generated pores, either in organic films or in lipid vesicles has been widely studied (Debregeas et al., 1995 \& Sandre et al., 1999). These synthetic materials are quite different in many aspects from the current plasma membranes of the live cell. Especially, they are quite inhomogeneous in distribution of viscosity, having a different elastic modulus and three-dimensional shape, and lastly have a different lipid composition. Meanwhile the previous investigation suggests that the viscosity of the films plays a very important role in opening and closing dynamics of the perforated holes (Sandre et al., 1999). If viscous dissipation is associated with the generation of transient pores dominates the inertia due to the very high viscosity, the leak out or extrusion of the cytosolic components slows down; the pore reaches the size up to $10 \mu \mathrm{m}$. The immersion of vesicles in a viscous environment allows visualization of transient pores in a membrane stretched by intense illumination or laser irradiations. Therefore, we have adapted a simple model to describe the dynamics of holes in the plasma membrane of live cells by an analogy with the opening of holes in viscous suspended long-chain polymer films and the dynamics of transient pores in stretched giant unilamellar vesicles (Sandre et al., 1999). The growth law of the hole is derived from a transfer of surface energy into viscous losses:

$$
4 \pi \eta d \frac{d r}{d t}=2 \pi r f
$$

where $\eta d$ is the surface viscosity, and $f$ is the surface tension acting on the film. The growth of pores in tense vesicles is more complex, because (i) the tension of the membrane relaxes to zero as a pore expands, and (ii) the line tension cannot be neglected. It is the driving force to close the pores. Including these two effects, we write the driving force $f$ per unit length of the hole as

$$
f=\sigma_{0}\left(1-\frac{r^{2}}{r_{c}^{2}}\right)-\frac{S}{r}
$$

where $\sigma_{0}$ is the membrane tension before the opening of the hole and $r_{c}$ is the maximum radius of the hole. The first term describes that the surface tension decreases linearly with the area of the pore. The second term is the restoring force that tends to close the pore because of the line energy $S$ per unit length.

Following the model, we are proposing the dynamics of live-cell plasma membrane associated with perforation of a hole with an initial radius of $r_{i}$, with further increment, closing and finally complete sealing of hole in the ruptured plasma membrane (Fig. 11). In a period of opening of the hole, the dynamics could be described in terms of a relaxation of surface tension, which is the plasma membrane tension before the perforation, due to the size increment of the hole. The growth of a hole is ruled by Eq. (1) with $f \approx \sigma_{0}\left(1-r^{2} / r_{c}^{2}\right)$. This leads to:

$$
\ln \frac{r}{r_{i}}-\frac{1}{2} \ln \frac{r_{c}^{2}-r^{2}}{r_{c}^{2}-r_{i}^{2}}=\frac{t}{\tau}
$$

where $\tau^{-1}=\sigma_{0} / \eta d$.

With opening of a hole at the radius $r$, extending to, in the presence of surface tension leads to an extrusion of the intracellular contents (Fig. 8(c)); finally reducing with the relaxation of the surface tension to zero. The increase in the radius as well as relaxation of the tension is 

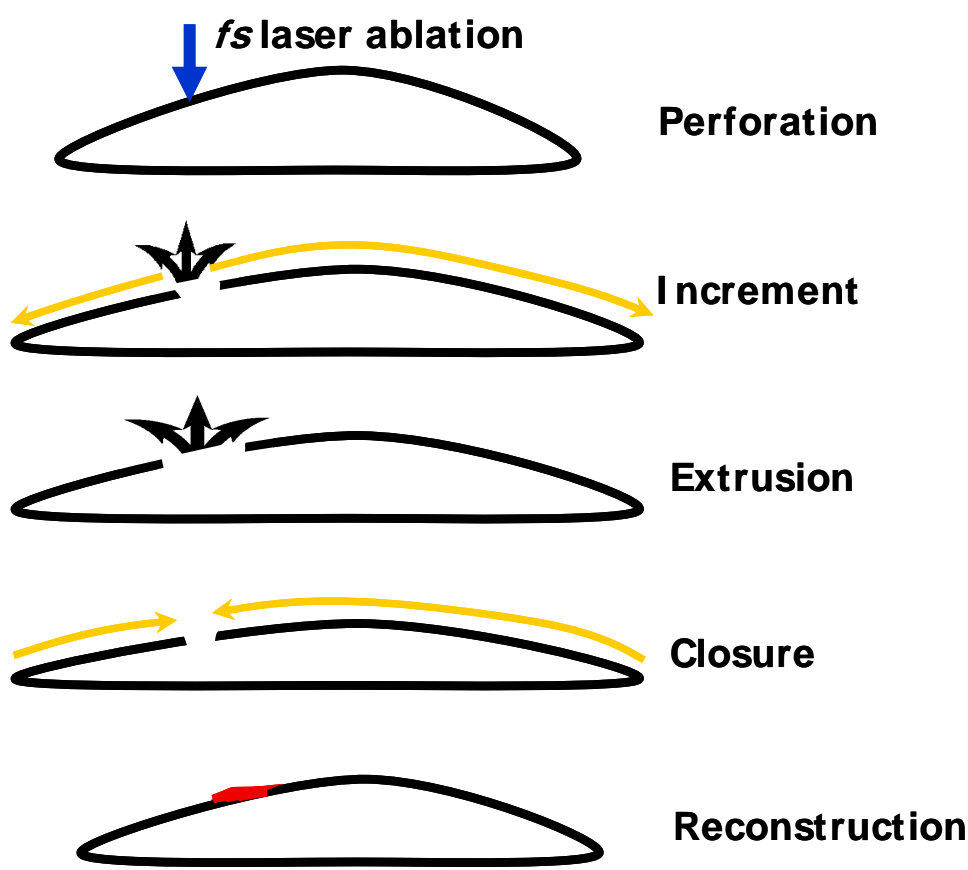

Fig. 11. Schematic diagram for the self-healing process after the fs- laser irradiation on livecell plasma membrane. Plasma membrane healing process consists of the following four successive processes. Opening: when the plasma membrane is ablated by fs-laser, a submicron-sized hole was created on the membrane surface. Increment: due to the presence of surface tension of the films, which consist of plasma membrane and cytoskeleton network such as F-actin, the radius is increased with radial flow of the plasma membrane. Extrusion: with growth of the hole size, the cellular contents extrude with a relaxation of the initial surface tension. Closure: after reaching the maximum radius of the hole, the restoring force for the surface line energy dominates the dynamics to reduce the radius of the hole. Sealing: Finally the cellular structures come close to each other and support the closure of ruptured hole where the intracellular supporting networks as well as the lipid molecules have been reconstituted completely.

associated with the domination of restoring force of the hole due to the increment of line energy $S$ per unit length. This finally induces the closure of the transient hole in order to reduce the line energy. Taking Eq. (1) into account, with the restoring force $f=-S / r$, the closing speed shall be approximately described as following:

$$
V=-\frac{d r}{d t}=\frac{S}{2 \eta d}
$$

Based on the above simple model, the dependence of the radius $r$ of the hole on time-lapse $t$ was fitted in both region of opening and closing of the holes using Equations (3) and (4), respectively. It is quite appealing that the observed temporal behavior of the hole radius is fairly well fitted with the parameters of $r_{i}, r_{c}$, and time constant $t$ of $0.3 \mu \mathrm{m}, 3 \mu \mathrm{m}$ and $12 \mathrm{sec}$, 
respectively. In the first order of approximation, the estimated speed of plasma membrane in opening region is found to be $0.2 \mu \mathrm{m} / \mathrm{sec}$. For the closing period, the fitting of the experimental data results in the speed of about $0.007 \mu \mathrm{m} / \mathrm{sec}$.

For comparison, the opening time constant and closing speed for a synthetic unilamellar vesicles made of 1,2-dioleoyl-sn-glycero-3-phosphocholine (DOPC) adhering on the substrate were reported to be $0.8 \mathrm{sec}$ with $r_{i} \sim 1.3 \mu \mathrm{m}$ and $r_{c} \sim 8.0 \mu \mathrm{m}$ and $1.8 \mu \mathrm{m} / \mathrm{sec}$, respectively (Sandre et al., 1999). It is remarkable to compare the closing speed $(S / 2 \eta d)$ of the live cell membrane, which is found to be 250 times slower than the synthetic vesicles. And also, the comparison on the time constant responsible for the opening of hole indicates that the opening process observed from live cell membrane is much slower than that for unilamellar stretched vesicles. These differences might be explained in terms of higher surface viscosity and/or the lower surface tension as well as lower surface line energy in the live cells. Without any exact physical constants for live cells including the above parameters, it is difficult to give any conclusive remarks on the dynamics observed from the current work. Assuming the viscosity of the viscous films on the surface of live cell and its energy loss per unit length to be curved at the edge of the holes is not quite different from those for the previous synthetic vesicles, however, the films in the flow field associated with the hole closing of the live cell must be of two orders of magnitude thicker than that of a synthetic unilamellar vesicles. This interpretation is contrary to the fact that the thickness of cellular membrane based on the fluid mosaic model should be of same order of magnitude as that of the synthetic one (Singer \& Nicolson, 1972). It's a well known fact that the cytoskeleton bound to membrane is a deformable intracellular support network made of interconnected filamentous biopolymers and its main constituent is the network of helical actin-filaments (F-actin) (Engelman, 2005; Singer \& Nicolson, 1972). So, it is reasonable to suppose that the nature of the films composed with membranes itself and the bound cytoskeleton structure due to its relatively long filament half-life of order of $10^{-1} \sim 1 \mathrm{~min}$, must be considered as parameters to explain the live cell membrane dynamics induced by the current ultrafast perforations (Oliver et al., 2005). In other words, the presence of the cytoskeleton bound to cell membrane results in the increment of effective thickness in the flow field of live cell membrane for hole growth and closure processes. In fact, typical thickness of actin cortex in motile osteoblasts was reported to be the order of $10^{-1} \sim 1 \mu \mathrm{m}$ (Oliver et al., 2005). The rather thick intracellular supporting network compared to a unilamellar membrane of synthetic vesicles should play an important role in the growth and closure dynamics of transient hole in the live cells and thus results in much higher surface viscosity of the films.

Finally, we would like to discuss some explanations for the irregular features observed from the topographical studies during the self-healing process (Fig. 8(f)). The topographical features were maintained for quite a long period even after the disappearance of optical signature of presence of holes on the cell membranes. The long lasting irregular ruffled structures of the membrane are likely to be associated with the final reconstruction of live cell membrane, to that of similar nature as present at the initial stages. Following the previous interpretations on the nature of films in the dynamic hole opening and closing behavior, the intracellular support network as well as the lipid molecules have been not reconstructed completely even though the cellular structures get close enough to do so. The chemistry and physics associated with the reconstruction process is still far from its complete understanding. We can suppose that a non-homogeneous reconstruction process at a relative slow reconstruction speed may result in the generation of irregular topographies at 

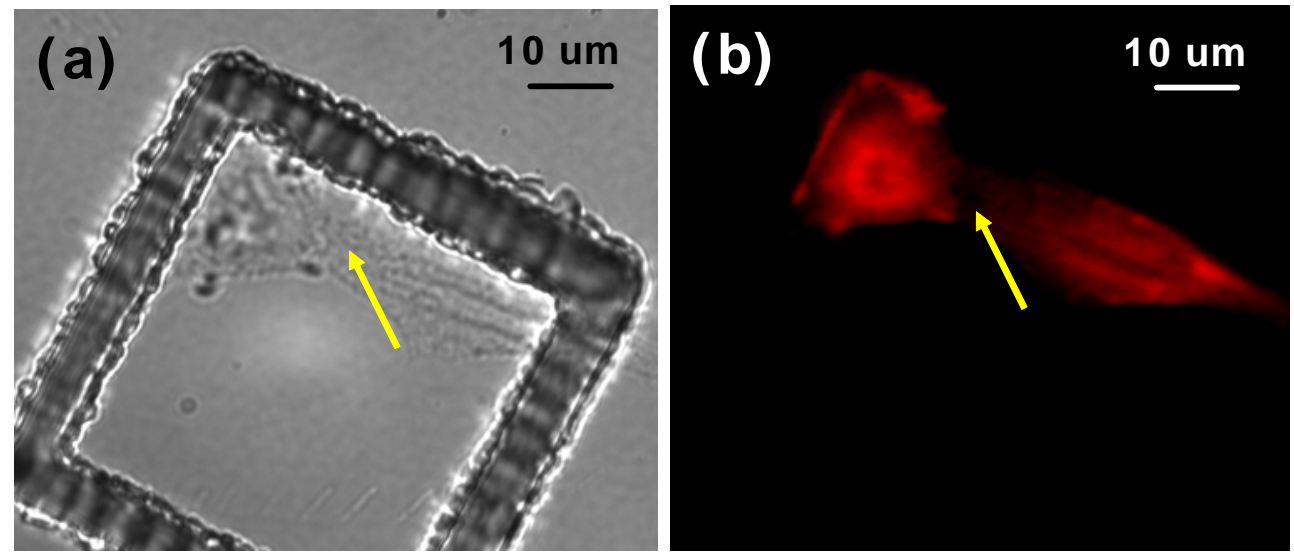

Fig. 12. Bright field (a) and fluorescence image (b) of injured cell stained with Alexa fluor 635 Palloidin (Molecular Probe) to visualize the distribution of F-actin. The cells were fixed and stained after completing the hole closing process under bright field images. The emission intensity near to the ablated region (arrowed) is less than that for other parts. The hollowed black area in the bright-field image (a) is a remnant of fs-laser ablated region, which was processed on the substrate as a guideline before seeding of the cells.

the edge of colliding films on the surface of cells. In order to obtain qualitative information concerning on this supposition, the cells just after completing the closing process under bright field images were stained to know the actin network distribution. As shown in Fig. 12, the fluorescence image shows the lower intensity of Alexa Flour 635 Palloidin expression near to the perforated region rather than other parts of the cells. This observation partly supports our proposition that the reconstruction process of F-actin network might results in several tiny slices of lipid during sealing and interconnecting the dissected cell membrane at the final stage of self-healing process. Another possible interpretation on the observations might be the self-healing process of damaged surface membrane of live cells such as urchin's egg and muscle cell by, so called, surface precipitation reaction mechanism proposed several tens of years ago (Heilbrunn, 1956; Gonzalez-Serrators et al., 1996). If this is the case, the piece of the lipid assembly should be together spontaneously to recover the injured surface membrane of live cells and resulted in the surface irregularity detected with only AFM techniques as shown in Fig. 8 (e)-(f). Even though no any conclusive remarks on the nature of the final reconstruction process of the damaged surface of the live cells will be given with the available information on the process, the irregular features observed should be strongly associated with reconstruction of not only the plasma membrane itself but also the actin network as an cytoskeleton structure of the live cells.

\section{Ultrafast laser- assisted optoperforation of primary retinal blood vessels}

\subsection{Experimental techniques and materials}

Furthermore, these extensive studies on single cell and plasma membrane dynamics open up the new scenarios, to apply this technology in a real surgical world, such as retinal blood vessel occlusions. 
Experimental porcine eyes obtained from a local abattoir were deposited in ice cold Hanks's Balanced Salt Solution (HBSS) and transported to the laboratory on ice $\left(4{ }^{\circ} \mathrm{C}\right)$ within $1 \mathrm{hr}$ after the slaughter. After a precise incision of the porcine eyes behind the ora serrata, cornea and lens, the retina tissues were carefully peeled off from pigmented epithelium and put it onto the Whatmann 2 paper. Before proceeding further for the fs-laser irradiations, the retina segments with size of about $3 \times 5 \mathrm{~mm}^{2}$ were prepared and to closely mimic the in vivo retinal conditions and to prevent the drying off the samples, we covered the tissues with 100 $\mu l$ of vitreous humour during the experiments.

The fs-laser system used in this study consists of a regenerative amplified Ti: Sapphire $(\lambda=$ $810 \mathrm{~nm}$ ) laser, delivering a 150-fs pulse at a repetition rate of $1 \mathrm{kHz}$ (Quantronix, USA). The laser beam is focused on the retina surface with an objective lens of N.A. 0.4 and the estimated laser beam diameter at the retinal surface is about $1.3 \mu \mathrm{m}$. Similar to the previous setups, the retina tissues are mounted onto a motorized XYZ translation stage, which is used to manipulate the samples to expose a fresh area of tissue to each laser pulse (Fig. 13). The laser fluence used in the current work is in the range of $1.4 \mathrm{~J} / \mathrm{cm}^{2} \sim 99.4 \mathrm{~J} / \mathrm{cm}^{2}$. With 13 different porcine eyeballs, 20 - 25 laser treatments for each eye ball were conducted at intervals of $100 \mu \mathrm{m}$ on the surface of retinal blood vessels. Blood vessels used in the experiments were primary in nature and were classified on the basis of the location and the

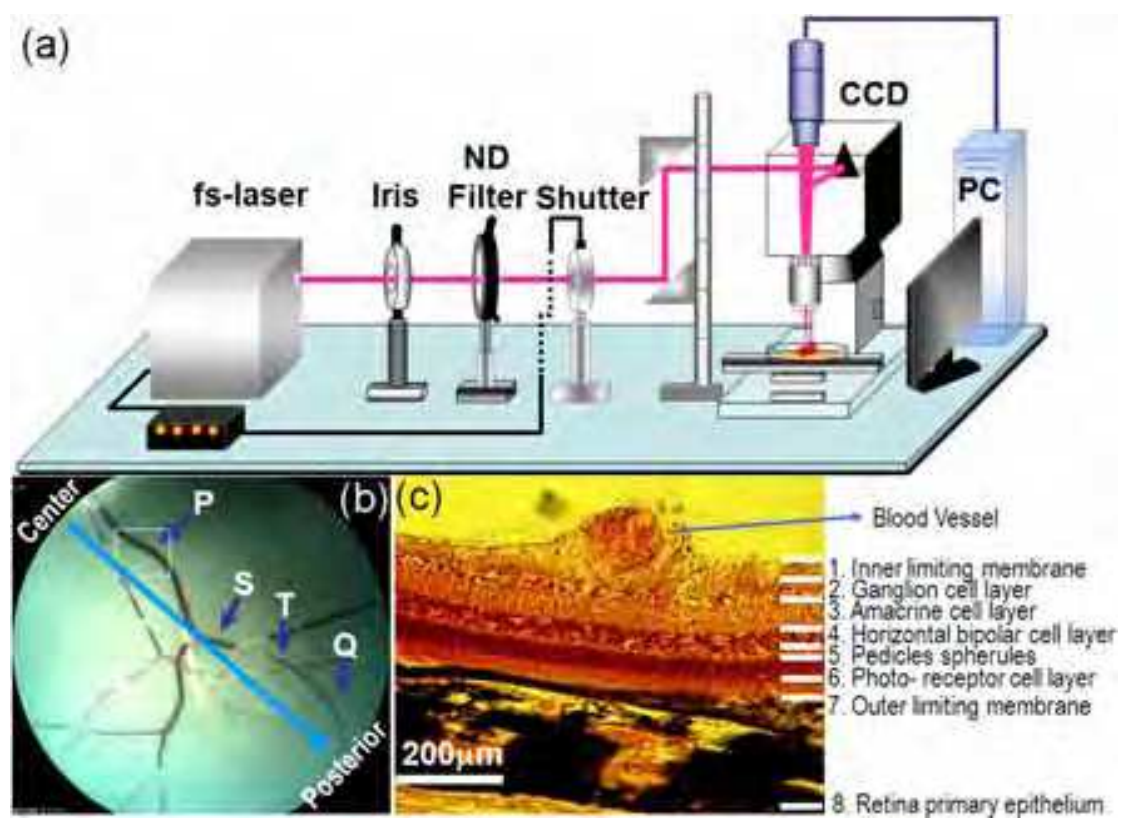

Fig. 13. (a) Schematic diagram of the experimental setup for fs-laser treatment of porcine retina blood vessels (b) The image from a fundus camera (Carl Zeiss Meditec, AG, Germany) shows the organization of blood vessels following the direction of blood flow from the center of the retina, i.e., the fovea region, to the peripheral retina: Key: $\mathrm{P}=$ Primary, $\mathrm{S}=$ Secondary, $\mathrm{T}=$ Tertiary and $\mathrm{Q}=$ Quarternary Blood Vessels. (c) The inset shows a typical cross-sectional image of the primary blood vessel, including the underlying multilayered structure of the porcine retina. 
diameter of the vessel. All the experiments were conducted under a single-shot configuration by using a mechanical shutter with an opening time constant of $0.6 \mathrm{~ms}$, which is synchronized with the laser, allows an accurate delivery of single pulse- irradiations into the samples. (Sidhu et al., 2009)

After optoperforation of blood vessel epithelium, the location of each laser lesion was carefully mapped. Just after laser treatment, the retina segments were immersed and fixed in $2 \% p$-formaldehyde at $4{ }^{\circ} \mathrm{C}$ for one hour. In next step, the retina tissues were submersed in $30 \%$ sucrose for about one hour to retain the cell shape. The fixed and retained samples were embedded in Jung's Tissue Freezing medium for an hour at room temperature and later on transferred to a refrigerator at -20 oC for 4-6 hrs. Transverse cryo-sections with a thickness of $20 \mu \mathrm{m}$ (Microtome, LEICA) were made of the lesion area at $-15{ }^{\circ} \mathrm{C}$. The slices were stained with Haemotoxylin and Eosin Red $(\mathrm{H} \& \mathrm{E})$ to assay the laser lesions at the peripheral Inner Limiting Membrane (ILM : upper layer of cells covering the entire retina surface along with the blood vessels) and the blood vessels epithelium of the retina tissue. In order to determine the percent probability of damage for the blood vessels, we examined about 1002 sampled (6 times) at various laser fluences in the range of $1.4 \mathrm{~J} / \mathrm{cm}^{2}-99.4$ $\mathrm{J} / \mathrm{cm}^{2}$.

\subsection{Results}

With changing laser fluence from $1.4 \mathrm{~J} / \mathrm{cm}^{2}$ to $99.4 \mathrm{~J} / \mathrm{cm}^{2}$, we produce a series of lesions on the blood vessel walls with a single fs-laser pulse. Laser irradiation on the surface of the retinal blood vessels with the fluence higher than about several tens of $\mathrm{J} / \mathrm{cm}^{2}$ results in an apparent disruption of the vessel walls, followed by a continuous outflow of blood within 2 $\sim 3$ seconds, as shown in Fig. 14. The red color of the vessels fades out after about 5 minutes. After 10 minutes, the bleeding is almost stopped. However, at lower laser fluence, the bleeding is stopped within one minute after extrusion of a little blood. (Sidhu et al., 2009)

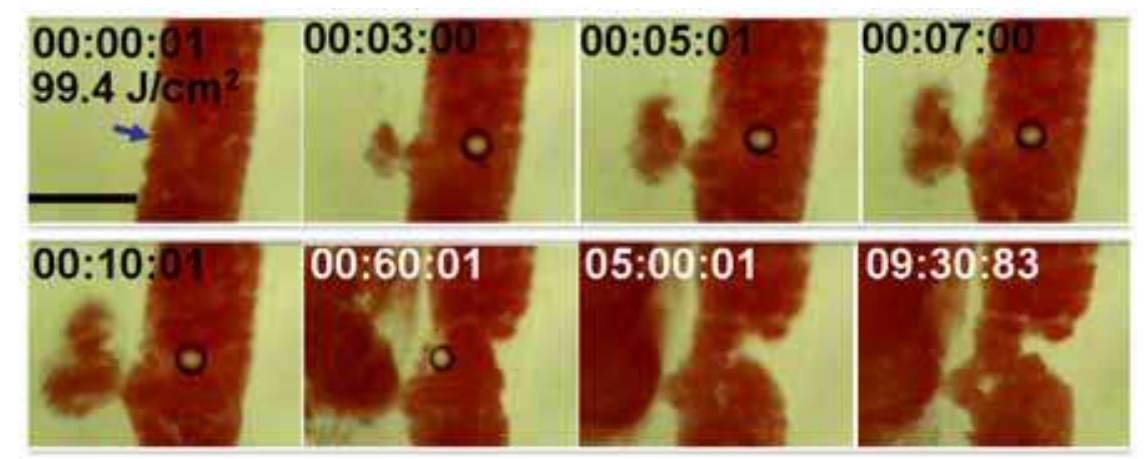

Fig. 14. Captured optical images of blood flow from a retinal blood vessel after exposure to a single fs-laser pulse at $810 \mathrm{~nm}$ with time scale from 0 to $10 \mathrm{~min}$. The laser beam is focused at the position indicated by the arrow head. Just after laser irradiation, a continuous outflow of blood is observed for $2 \sim 3$ seconds. The scale bar is $100 \mu \mathrm{m}$.

To examine the blood vessels of a porcine retina after fs-laser irradiation, we sequentially sectioned the retina segment with primary blood vessels with a thickness of $20 \mu \mathrm{m}$. Figure 15 represents histological images of the sectioned slices. The laser irradiations at lower 
fluence cause damage on the surface of the blood vessels with a low probability. At higher laser fluence, however, almost all the retinal segments exhibit apparent disruption of the ILM, which mainly consists of a Glial cell layer. Apparent disruptions of the ILM in the sectioned slices of the retina treated at $14.2 \mathrm{~J} / \mathrm{cm}^{2}$ could be observed in the first and the fifth slices without any visible alterations in other sequential slices. No apparent disruption in the

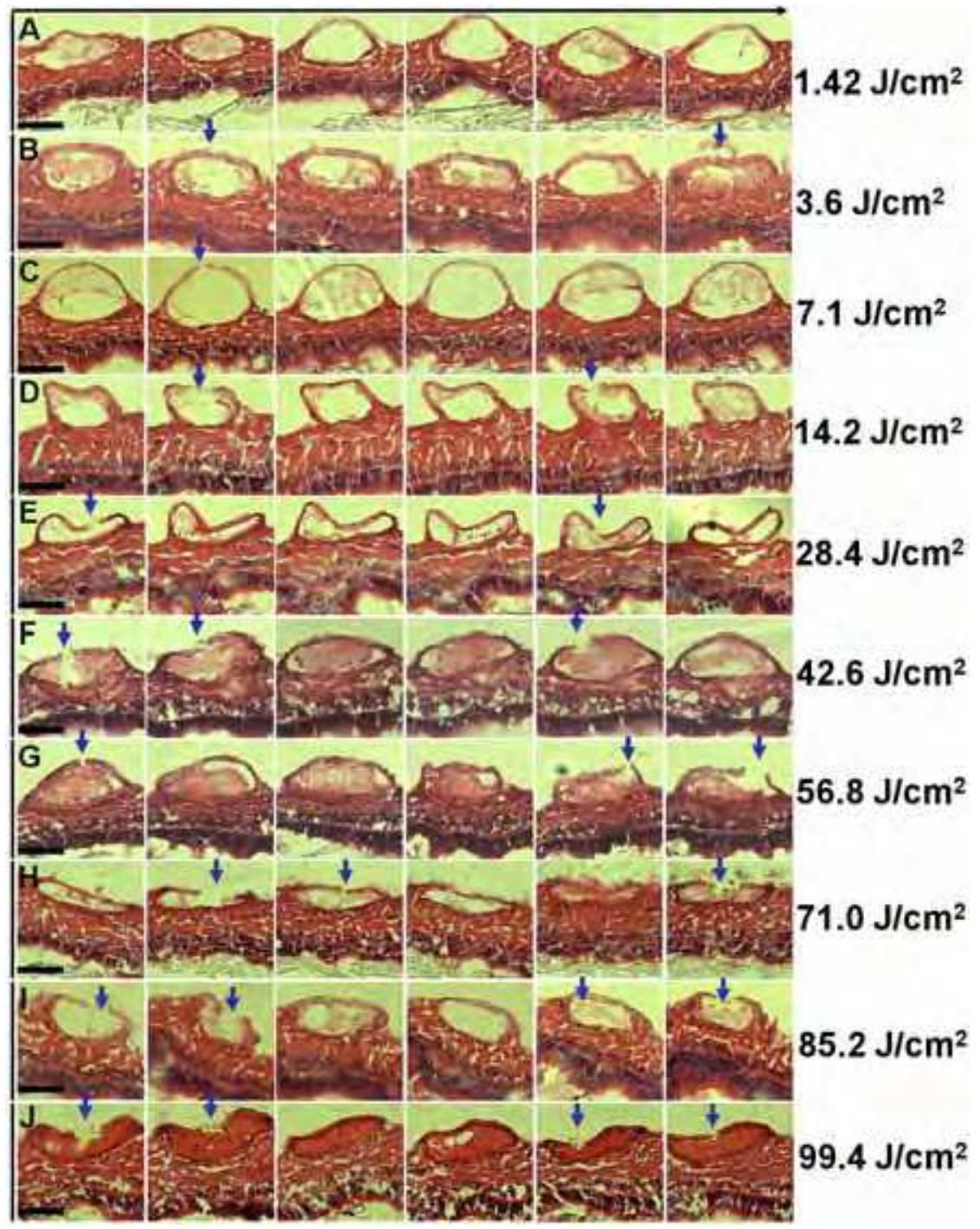

Fig. 15. Sequential sections of a blood vessel lesion caused by a single fs- laser pulse irradiation. The thickness of the sectioned slices is $20 \mu \mathrm{m}$. Figures on the right-hand side represent laser fluence. The arrow head indicates a lesion observed on the blood vessels. The scale bar is $100 \mu \mathrm{m}$. 
surface of the blood vessels endothelial layer and no bleeding after laser irradiation were observed. If the slice thickness of $20 \mu \mathrm{m}$ in the sectioning procedure is considered, the interval between slices with an apparent disruption is $100 \mu \mathrm{m}$, which is quite consistent with the distance between laser-irradiated points on the retina tissue. This observation suggests that from the histological analysis, the lateral damage of the retina upon fs-laser exposure is limited to $20 \mu \mathrm{m}$ range. In fact, the diameter of the laser lesions on the surface of treated retina, examined via scanning electron microscopy, is found to be less than $4 \mu \mathrm{m}$ (Fig. 16). With increasing laser fluence to $28.4 \mathrm{~J} / \mathrm{cm}^{2}$, single-pulse laser irradiation induces complete optoperforation on the blood vessel wall. With further increment in the laser fluence to 52.6 $\mathrm{J} / \mathrm{cm}^{2}$, the frequency of sequential slices with observed prominent damage on the blood vessels increases. This reveals that the diameter of the induced lesion is larger than $20 \mu \mathrm{m}$ under these rather higher laser fluences. The data suggest that up to $85.2 \mathrm{~J} / \mathrm{cm}^{2}$, there is no observable alteration in underlying retina cell layers, except for disruptions in both the blood vessel walls and the ILM. Under a higher fluence of $99.4 \mathrm{~J} / \mathrm{cm}^{2}$, however, a slight distortion is observed in the underlying tissue (Fig. 15(J)). (Sidhu et al., 2009)
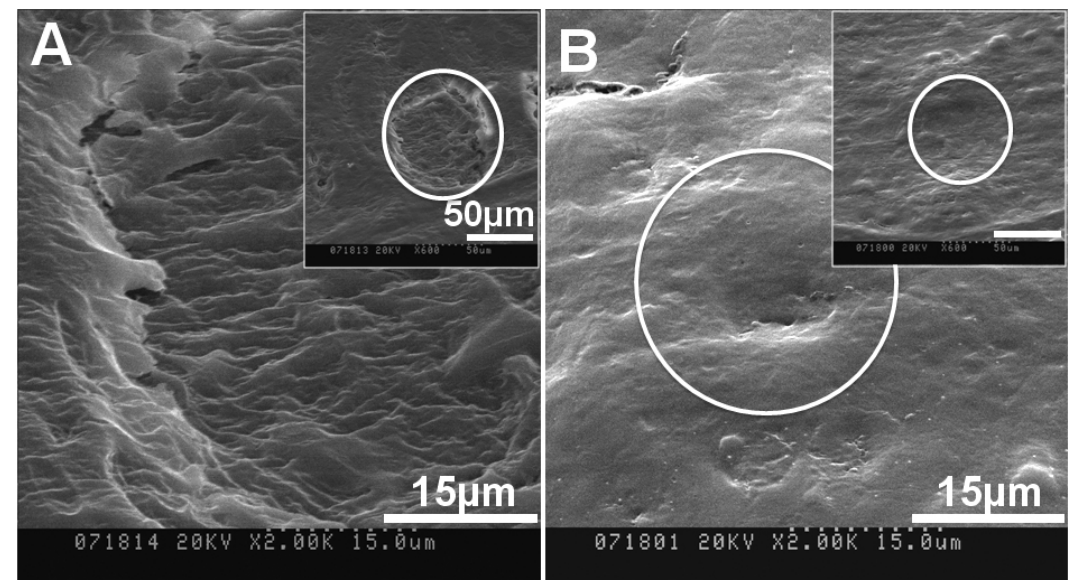

Fig. 16. Scanning electron microscopic images for a laser-irradiated retina surface (white circles) with two different laser fluences $99.4 \mathrm{~J} / \mathrm{cm}^{2}(\mathrm{~A})$ and $7.1 \mathrm{~J} / \mathrm{cm}^{2}(\mathrm{~B})$. The insets show SEM images with lower magnification.

The cryosections of the retina segments irradiated with a single-shot ultrafast laser were tentatively grouped into three types of lesions: no change, ablation only at the ILM and optoperforation of blood vessel walls (Fig 17). Correlation statistics of these different types of lesions is shown in Fig. 18 as a function of laser fluence. The optoperforation probability of blood vessels increased in the fluence range of $3.6 \mathrm{~J} / \mathrm{cm}^{2} \sim 99.4 \mathrm{~J} / \mathrm{cm}^{2}$. We determined the laser fluence to damage the ILM layer, found to be in the range between $1.4 \mathrm{~J} / \mathrm{cm}^{2}$ and 3.6 $\mathrm{J} / \mathrm{cm}^{2}$. However, perforation of the blood vessels is apparently caused by femtosecond-laser irradiation with a higher fluence between $3.6 \mathrm{~J} / \mathrm{cm}^{2}$ and $7.1 \mathrm{~J} / \mathrm{cm}^{2}$. If the percent probability at low fluence is extrapolated, the fluence at zero probability of alteration of the ILM and the perforation of the blood vessels are estimated to be $2.19 \pm 1.08 \mathrm{~J} / \mathrm{cm}^{2}$ and $5.85 \pm 1.49 \mathrm{~J} / \mathrm{cm}^{2}$, respectively. (Sidhu et al., 2009) 

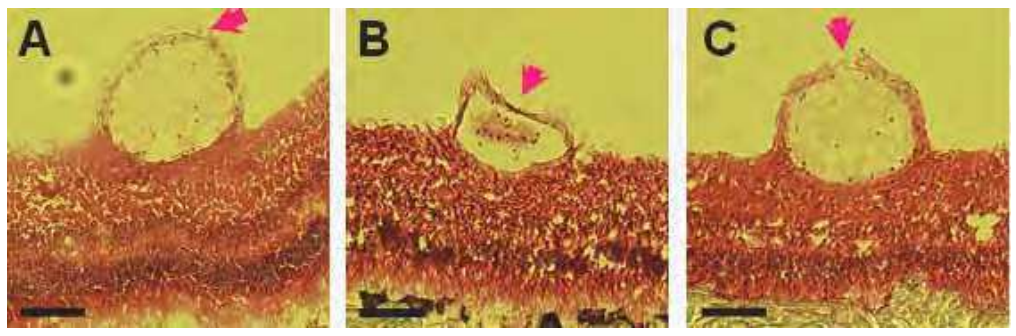

Fig. 17. Representation of retinal segments irradiated with a single-shot ultrafast lasers. They were tentatively grouped into three types of lesions: A. No change, B. The ablations at the ILM and $\mathbf{C}$. The optoperforation of blood vessel walls. The arrow head indicates the point of irradiations on the blood vessels.

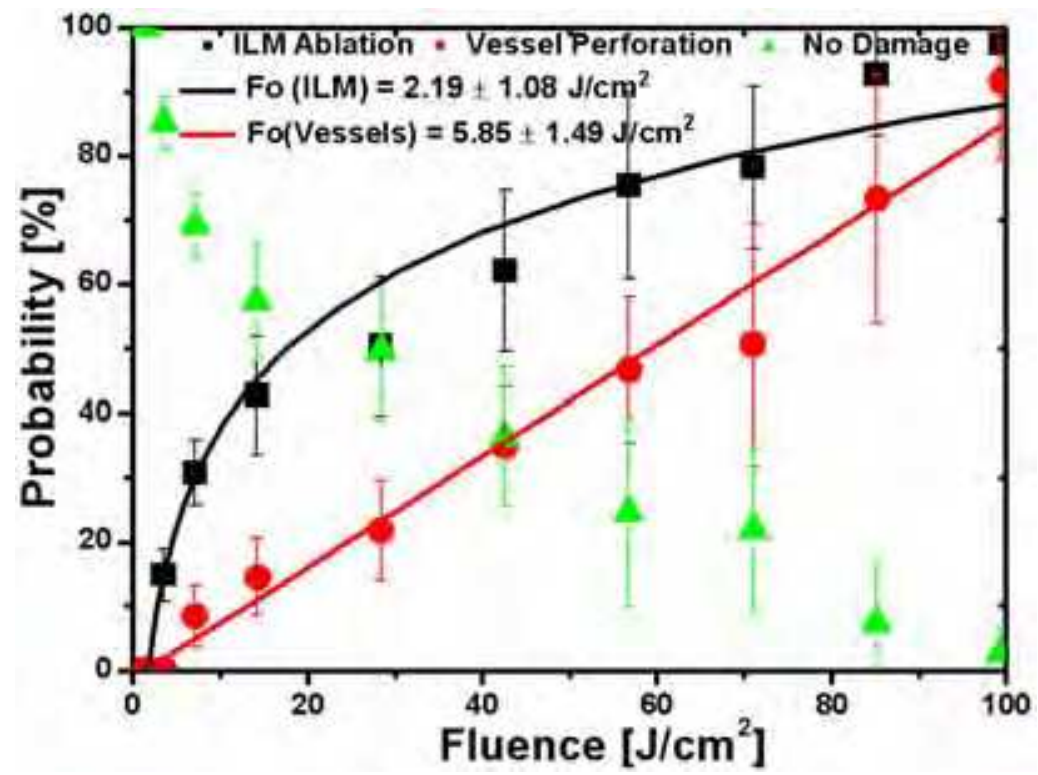

Fig. 18. Linear plot of the percent probability for inner limiting membrane (ILM) damage (solid rectangles) and vessel perforation (solid circles) as a function of the laser fluence. The ablation threshold fluence for ILM and blood vessels was found to be $2.19 \pm 1.08 \mathrm{~J} / \mathrm{cm}^{2}$ and $5.85 \pm 1.49 \mathrm{~J} / \mathrm{cm}^{2}$, respectively. With increasing fluence, the percent probability of blood vessel perforation monotonically increases. The lines represent an extrapolation to determine the ablation thresholds for perforation of retinal primary blood vessels and for ILM damage of a porcine eye.

\subsection{Discussion}

Recent development in advanced laser technology transiently facilitates to perform transaction, ablation, and coagulation of tissues via delivery of laser irradiation into a small focal volume are providing an attractive possibilities for new laser surgical technologies. The laser beam is a potential candidate that has already undergone a multi-center clinical 
trial to evaluate the feasibility for its use in vitreoretinal surgery (Schastak et al., 2007). Limited precision and significant damage by lasers with relative long pulse durations does not allow partial or selective tissue ablation with high precision. If such damage is to be overcome, infrared laser sources, such as $\mathrm{CO}_{2}$, Er:YAG and Holmium:YAG lasers, have undergone several trials via optical fiber delivery in intraocular surgery. However, apparent collateral damage to surrounding tissue due to significant thermal and shockwave effects have been reported (Paula- Yu et al., 2006).

Laser ablation of tissues could be described using either an optical breakdown model or a thermal confinement models. The optical breakdown model considers plasma formation and subsequent shock wave formation, cavitation, and tissue disruption. The thermal confinement model recognizes the competing thermal effects of the vaporization of water driving an explosive ablation and thermal diffusion leading to collateral damage. This model accounts for the observation that collateral damage is limited if the pulse duration is less than the thermal relaxation time of the ablated tissue volume (Vogel et al., 2003; Apitz et al., 2005).

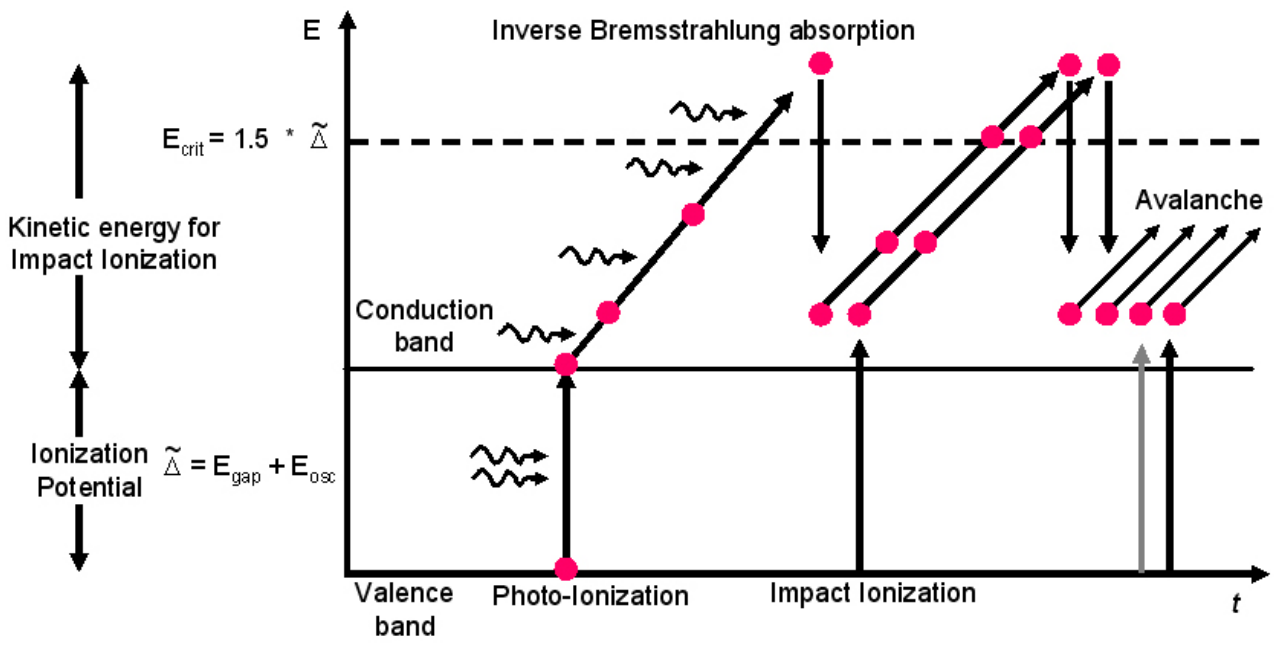

Fig. 19. Interplay of photoionization, inverse Bremsstrahlung absorption, and impact ionization in the process of plasma formation. Recurring sequences of inverse Bremsstrahlung absorption events and impact ionization lead to an avalanche growth in the number of free electrons. (Vogel et al., 2005)

The process of plasma formation through laser induced breakdown in transparent biological media is schematically depicted in Fig. 19. It essentially consists of the formation of quasifree electrons by interplay of photoionization and avalanche ionization. It's a well known fact that the optical breakdown threshold in water is very similar to that in ocular and other biological media (Docchio et al., 1986). Irradiation by an intense ultrafast laser beam further leads to multiphoton excitation of a target material. The absorbed energy might be transported to the electrons without thermal diffusion to adjacent material because the pulse width is shorter than the vibrational relaxation time constant of several picoseconds. As a result, thermal damage on the surrounding tissues could be minimized, and the biological tissue remains unaffected by the subsequent photoinduced mechanical shock process. This 
effectively renders the fs-laser surgical process non-thermal. The formation of a high density of free electrons could result in a local plasma formation in the targeted materials. This hot plasma formation results in a permanently damaged region, even inside a cell with a submicron size (Vogel et al., 2005). Furthermore, a previous on tissues like the corneal stroma revealed that the ablation threshold fluence decreased with increasing pulse width of the applied laser (Preuss et al., 1995). These uniquely show that ultrafast lasers can be utilized for precise treatment of tissues while minimizing any apparent thermal damage or shock pressure to biological tissues (Kohli et al., 2005). The results illustrated in the current work made the above hypothesis true for the retinal tissues, where retinal blood vessels were selectively perforated with wide range of laser fluence $\left(1.42 \sim 99.4 \mathrm{~J} / \mathrm{cm}^{2}\right)$ with an ultra fast laser in near infra red region.

From the past literature values for the ablation thresholds for various tissues, including the corneal stroma, axons, the eye's anterior chamber, and hard tissue (under a single-shot configuration, as in current work), the ablation threshold of the corneal stroma for an ultrafast laser is in the range of $1 \mathrm{~J} / \mathrm{cm}^{2}$ to $2 \mathrm{~J} / \mathrm{cm}^{2}$. Meanwhile, the ablation threshold for axons of C. elegans is reported to be about $4.4 \mathrm{~J} / \mathrm{cm}^{2}$. It is of great interest to note that the value for the femtosecond laser ablation threshold of the ILM of the porcine retina, $2.19 \pm$ $1.08 \mathrm{~J} / \mathrm{cm}^{2}$ as determined in the current work, is in the same range of reported values for the soft tissues. It is also interesting to compare the ablation threshold of the retina upon irradiation by a femtosecond laser to the values for irradiation with an ultraviolet (UV) laser with a nanosecond pulse width, including ArF excimer lasers and higher-harmonic Nd:YAG lasers. The ablation threshold is reported to be in the range of between $0.6 \mathrm{~J} / \mathrm{cm}^{2}$ and 1 $\mathrm{J} / \mathrm{cm}^{2}$ when irradiating single-pulsed UV light into the retina tissue, which is slightly lower than that for femtosecond laser ablation threshold. Considering the remarkable difference in the linear optical absorption coefficients of the retina tissue in the UV and the NIR ranges, it is reasonable to suppose that an ultrafast laser operating in the NIR region would be able to ablate the ILM layer in the retina with much lower deposited energy per unit volume compared to UV nanosecond lasers. The perforation threshold of the underlying primary retinal blood vessels $\left(5.85 \pm 1.49 \mathrm{~J} / \mathrm{cm}^{2}\right)$ is significantly higher than the literature values.

The thickness of the ILM, which is essentially a basement membrane consists of retina müller cells, is only $6 \mu \mathrm{m}$ to $10 \mu \mathrm{m}$. The thickness of the ILM is thinnest at the fovea region of the retina. However, the thickness is larger at the posterior pole of retina (Hoerauf et al., 2006). Furthermore, the ILM is also present over the retinal blood vessels. If only the ILM is to be ablated selectively without any alterations in the underlying layers, the energy delivered by the laser irradiation must be confined in thin layers without any apparent diffusion of the deposited energy into other parts of the retina. To evoke this topic, we have examined the dependence of the ablation depth for transparent materials, like retinal tissue, on the laser fluence (Fig. 20). If there is high free electron density due to optical absorption processes, we suppose that the underlying mechanism for the ablation by fs-laser irradiation is not directly governed by the optical and the electronic properties of the materials. Even if the absorption mechanism of the NIR fs-laser is dependent on the optical band gap of each material, two different slopes under fs laser irradiation have already been reported for metals, semiconductors, and dielectrics (Nolte et al., 1997; Furusawa et al., 1999). For a lower laser fluence, $\mathrm{F}$, the ablation depth can be described by the expression $\mathrm{L}=\delta \ln \left(\mathrm{F} / \mathrm{F}_{\mathrm{th}}{ }^{\delta}\right)$ ), where $\delta$ is the optical penetration depth and $\mathrm{F}_{\mathrm{th}}\left({ }^{\delta}\right)$ is the threshold laser fluence of ablation [Preuss et al., 1995, Jia et al., 2006]. A fit to the experimental data results in $\mathrm{F}_{\text {th }}^{\delta}=2.2 \pm 0.9$ 
$\mathrm{J} / \mathrm{cm}^{2}$ and $\delta=8.2 \pm 2.2 \mu \mathrm{m}$. It should be notified that the optical penetration depth is governed by a nonlinear optical transition, if multi-photon absorption plays an important role in photo-excitation of the materials. Therefore, the optical penetration depth estimated from the current work is difficult to reconcile with the literature value of the optical absorbance of retina tissue at a wavelength of $810 \mathrm{~nm}$. Due to the strong dependence of the multiphoton absorption on the energy density, the value of $\delta$ should be relatively small. At any rate, it is of great interest to compare the observed optical penetration depth with the thickness of the ILM in the porcine retina. This comparison led us to propose that the energy delivered by femtosecond laser irradiation under the controlled laser fluence can be confined in the ILM layer, followed by a selective ablation of the layers only if the optical penetration depth of $8.2 \pm 2.2 \mu \mathrm{m}$ is comparable to the thickness of the ILM of the retina.

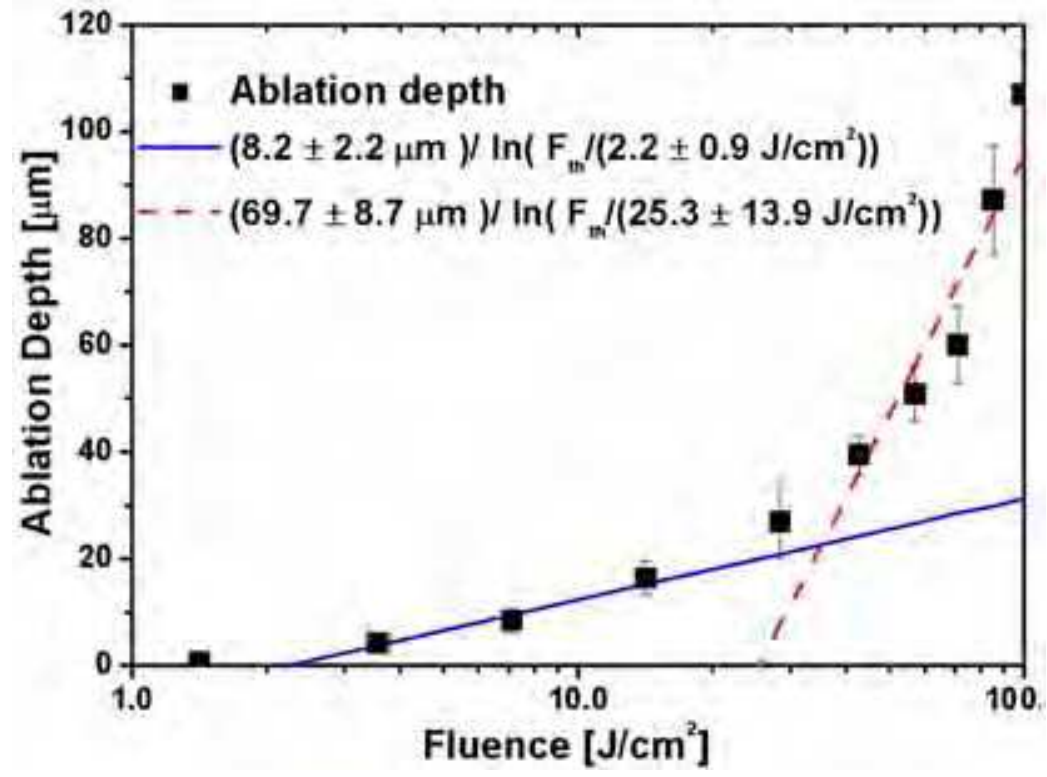

Fig. 20. The lesion depth of a porcine retina caused by fs-laser irradiation as a function of the laser fluence. The blue solid and the red dotted lines represent linear fit. About 300 sectioned slices from more than 10 eyeballs were examined for each laser fluence.

With increasing laser fluence, however, the mechanism underlying the retina ablation can no longer be expressed by the optical penetration depth. As shown in Figure 16, the retina surface treated with a high laser fluence of $99.4 \mathrm{~J} / \mathrm{cm}^{2}$ is very much roughened compared to the surface treated with a low fluence of $7.1 \mathrm{~J} / \mathrm{cm}^{2}$. Based on the changes in the slopes of the semi-logarithmic plot of the ablation depth as function of the fluence, we have supposed that at laser fluence higher than $25.3 \mathrm{~J} / \mathrm{cm}^{2}$, the electronic heat diffusion process plays an important role, even in an ultrafast laser ablation. The ablation depth in this region can be described with the expression of $\mathrm{L}=l \ln \left(\mathrm{F} / \mathrm{F}_{\mathrm{th}}{ }^{(l)}\right)$, where $l$ is the electronic heating depth, and $\mathrm{F}_{\mathrm{th}}{ }^{(l)}$ is the corresponding threshold fluence. The electronic heating depth and $\mathrm{F}_{\mathrm{th}}{ }^{(l)}$ are estimated to be $69.7 \pm 8.7 \mu \mathrm{m}$ and $25.3 \pm 13.9 \mathrm{~J} / \mathrm{cm}^{2}$, respectively, which means that the thickness of the retina tissue affected by fs-laser irradiation might be abruptly increased for 
the laser fluence higher than $25.3 \mathrm{~J} / \mathrm{cm}^{2}$. As a result, we have to control the laser fluence very precisely to achieve a selective peeling of the ILM layer without any visible thermal damage being induced by the laser irradiation.

The probability of retina blood vessel damage shows a linear relationship with the laser fluence. With the progressive increase in the laser fluence, selective ablations of concerted retina layers even including primary blood vessels is possible without any apparent damage to the underlying layers of the porcine retina. The threshold fluence to perforate the walls of the primary blood vessels embedded in the porcine retina is estimated to be $5.85 \pm 1.49$ $\mathrm{J} / \mathrm{cm}^{2}$. If the ablation depth depends on the laser fluence as $\delta \ln \left(\mathrm{F} / \mathrm{F}_{\mathrm{th}}{ }^{(\delta)}\right)$, the thickness of the tissues ablated by a single-shot fs-laser pulse can be estimated to be $8.0 \pm 3.0 \mu \mathrm{m}$, by using the parameters of $\delta$ and $\mathrm{F}_{\mathrm{th}}{ }^{(\delta)}$ from this work. Meanwhile, the thickness of the tissues covering the primary blood vessels is tentatively determined to be about $25 \mu \mathrm{m}$ by examining the sectioned slices shown in Fig. 15. If the current interpretation for the ablation depth of the tissues by fs-laser irradiation is correct, the fluence to perforate the primary blood vessels should be about $46 \mathrm{~J} / \mathrm{cm}^{2}$. However, the ablation depth per pulse in the highlaser-fluence region should be described in terms of electronic heating depth with the relation of $\mathrm{L}=\delta \ln \left(\mathrm{F} / \mathrm{F}_{\mathrm{th}}{ }^{(l)}\right)$. With the parameters of $l$ and $\mathrm{F}_{\mathrm{th}}{ }^{(l)}$, we are able to estimate the fluence to fully perforate the primary blood vessels of the retina to be $36.2 \mathrm{~J} / \mathrm{cm}^{2}$. This value for blood vessel perforation is very close to the laser fluence at $1 / \mathrm{e}^{2}$ percent perforation probability, as shown in Fig. 18.

\section{Conclusion}

In summary, all the observations from the present work reveals that fs-laser irradiation on pollen walls to make an evident physical hole with an outside diameter of about $1 \mu \mathrm{m}$ well conserves the physiological state of the cell including its viability and pollen tube germination capability. Furthermore, from the successful delivery of foreign DNA into pollen through the hole reveals that the current method has an evident potential in the field of plant genetic engineering.

Topographical imaging as well as optical imaging of the plasma membranes led us to observe a self-healing process for live cells within several minutes of time after the fs-laser ablation on the live cells. A simple viscoelastic model for both the hole opening and closing process was found to be applicable to interpret its dynamics. The very slow dynamics could be explained in terms of high surface viscosity due to the presence of cytoskeleton network bound to the plasma membrane. The irregular feature in plasma topography observed in the final stage of the healing process might be due to a slice of the assembled lipid, which resulted from the reconstruction of not only the plasma membrane itself but also F-actin network as a cytoskeleton structure of live cells. Although two-dimensional plug flow model adapted in the current work fairly well interpret the experimental observations in macroscopically, the presence of transmembrane proteins, transbilayer interactions, and adhesion sites, etc., in addition to the bound cytoskeleton structure, produces a variety of restrictions on the flow dynamics of the plasma membrane through an alterations in many microscopic physico-chemical properties including thickness and hydrodynamic properties of the fluidic films.

We have developed a new method for elucidating more exact mechanism on the interesting topic of self-healing process based on ultrafast laser perforation of the plasma membrane of the animal cell. A mechanical stimulus to live-cell plasma membrane by the induced surface 
tension as well as surface line energy can be also applied by the current methods with high spatial resolution and unattainable speed of perforation. So interesting is the spatiotemporal characterization of the plasma membrane movement associated with the healing process that is closely related with the cell migration and transmission of the mechanical stimuli into biochemical signals, which might be mainly governed by cytoskeleton structure (Wang et al., 2005; Yamazaki et al., 2005; Supatto et al., 2005).

We have also successfully applied the current fs- laser technology to selectively perforate the retinal blood vessels without any apparent damage in the concerted retina layers. It provides a major breakthrough for the retinal vein occlusion therapy and removal of abnormal blood vessels (Choroidal Neovascularization $(\mathrm{CNV})$ ) grown during numerous retinal diseases.

\section{Acknowledgements}

This work was financially supported by the Ministry of Knowledge Economy of Korea and KRISS program.

\section{References}

Apitz, I. \& Vogel, A. (2005) Material ejection in nanosecond Er: YAG laser ablation of water, liver, and skin, Appl. Phys. A 81, 329-338

Aronen, T. S.; Nikkanen, T. O. \& Haggman, H. M. (2003). The production of transgenic Scots pine (Pinus sylvestris L.) via the application of transformed pollen in controlled crossing, Transgenic Res. 12, 375-378

Benkert, R.; Obermeyer, G. \& Bentrup, F. W. (1997). The turgor pressure of growing lily pollen tubes, Protoplasm 198, 1-8

Debregeas, G.; Martin, P. \& Brochard-Wyart, F. (1995). Viscous bursting of suspended films, Phys. Rev. Lett. 75, 3886-3889

Docchio, F.; Sachhi, C. A. \& Marshall, J. (1986) Experimental investigation of optical breakdown thresholds in ocular media under single pulse irradiation with different pulse durations, Lasers Ophthalmol. 1, 83-91

Engelman, D. M. (2005). Membrane are more mosaic than fluid, Nature 438, 578-570

Fernado, D. D.; Richards, J. L. \& Kikkert, J. R. (2006). In vitro germination and transient GFP expression of American chestnut (Castanea dentate) pollen, Plant Cell Rep. 25, 450456

Furusawa, K.; Takahashi, K.; Kumagai, H.; Midorikawa, K. \& Obara, M. (1999) “Ablation characteristics of $\mathrm{Au}, \mathrm{Ag}$, and $\mathrm{Cu}$ metals using a femtosecond: Ti Sapphire laser, Appl. Phy. A. 69(7), S359-S366

Gonzalez-Serratos, H.; Rozycka, M.; Cordoba-Rodriguez, R. \& Ortega, A. (1996). Membrane healing and restoration of contractility after mechanical injury in isolated skeletal muscle fibers of the frog, Proc. Natl. Acad. Sci. USA 93, 5996-6001

Greulich, K. O. \& Weber, G. (1992). The light microscope on its way from an analytical to a preparative tool, J. Microsc. 167, 127-151

Heilbrunn, L. V. (1956). The surface precipitation reaction, In: The Dynamics of Living Protoplasm 62-84, Academic, New York

Higashiyama, T.; Yabe, S.; Sasaki, N.; Nishimura, Y.; Miyagishima, S.; Kuroiwa, H. \& Kuroiwa, T. (2001). Pollen Tube Attraction by the Synergid Cell, Science 293, 1480-1483 
Hoerauf, H.; Brix, A.; Winkler, J.; Droege, G.; Winter, C.; Birngruber, R.; Laqua, H.; and Vogel, A. (2006) A Photoablation of inner limiting membrane and inner retinal layers using the Erbium: YAG-laser: An in vitro study, Lasers Surg. Med. 38(1), 52-61

Hoffmann, F. (1996). Laser microbeams for the manipulation of plant cells and subcellular structures, Plant Science 113, 1-11

Jeoung, S. C.; Kim, H. S.; Park, M. I.; Lee, J.; Kim, C. S. \& Park, C. O. (2005). Preparation of room-temperature photoluminescent nanoparticles by ultrafast laser processing of single-crystalline Ge, Jap. J. Appl. Phys. 44, 5278-5281

Jia, T. Q.; Chen, H. X.; Huang, M.; Zhao, F. L.; Li, X. X.; Xu, S. Z.; Sun, H. Y.; Feng, D. H.; Li, C. B.; Wang, X. F.; Li, R. X.; Xu, Z. Z.; He, X. K. and Kuroda, H. (2006) Ultravioletinfrared femtosecond laser-induced damage in fused silica and $\mathrm{CaF}_{2}$ crystals Phys. Rev. B 73 (5) 054105-1 - 054105-9

Kobayashi, N.; Rivas-Carrillo, J. D.; Soto-Gutierrez, A.; Fukazawa, T., Chen, Y.; NavarroAlvarez, N. \& Tanaka, N. (2005). Gene delivery to embryonic stem cells, Birth Defects Research (Part C) 75, 10-18

Kohli, V.; Elezzabi, A. Y.; \& Acker, J. P. (2005) Cell nanosurgery using ultrashort (femtosecond) laser pulses: applications to membrane surgery and cell isolation, Lasers Surg. Med. 37, 227-230

König, K.; Riemann, I.; Fischer, P. \& Halbhuber, K. J. (1999). Intracellular nanosurgery with near infrared femtosecond laser pulses, Cell. Mol. Biol. 45, 195-201

Krautwig, B \& Lörz, H. (1995). Cereal protoplasts, Plant Science 111, 1-10

Lee, Y. J.; Kim, D. H.; Kim, Y. \& Hwang, I. (2001). Identification of a signal that distinguishes between the chloroplast outer envelope membrane and the endomembrane system in vivo," Plant Cell 13, 2175-2190

Lovy-Wheeler, A.; Cardenas, L.; Kunkel, J. G. \& Hepler, P. K. (2007). Differential organelle movement on the actin cytoskeleton in lily pollen tubes. Cell Motil Cytoskeleton. 64, 217-232

Nelson, J. S. \& Berm, M. W. (1989). Laser application in biomedicine. Part II: Clinical applications, J. Laser Appl. 1, 9-20

Nolte, S.; Momma, C.; Jacobs, H.; Tünnermann, A.; Chichkov, B. N.; \& Wellegehausen, B. (1997) Ablation of metals by ultrashort laser pulses, J. Opt. Soc. Am. B 14, 2716-2722

Oliver, J. M.; King, J. R.; Mckinlay, K. J.; Brown, P. D.; Grant, D. M.; Scotchford, C. A. \& Wood, J. V. (2005). Thin-film theories for two-phase reactive flow models of active cell motion, Mathematical Medicine and Biology 22, 53-98

Parpura, V.; Haydon, P. G. \& Henderson, E. (1993). Three-dimensional imaging of living neurons and glia with the atomic force microscope, J. Cell Sci. 104, 427-432

Paula-Yu, K.; Miller, J.; Cringle, S. J.; \& Yu, D-Y. (2006) Experimental retinal ablation using a fourth-harmonic $266 \mathrm{~nm}$ laser coupled with an optical fiber probe, Invest. Ophthalmol. Vis. Sci. 47(4), 1587-1593.

Preuss, S.; Demchuk, A.; \& Stuke, M. (1995) Sub-picosecond UV laser ablation of metals, Appl. Phys. A 61, 33-37

Sandre, O.; Moreaux, L. \& Brochard-Wyart, F. (1999). Dynamics of transient pores in stretched vesicles, Proc. Natl. Acad. Sci. USA 96, 10591-10596

Schastak, S.; Yafai, Y.; Yasukawa, T.; Wang, Y. S.; Hillrichs, G. \& Wiedemann, P. (2007) Flexible UV light guiding system for intraocular laser microsurgery, Lasers Surg. Med. 39, 353-357

Senz, R. \& Miiller, G. (1989). Laser in Medicine, Ber. Bunsenges. Phys. Chem. 93, 269 -277 
Shen, N.; Datta, D.; Schaffer, C. B.; LeDuc, P.; Ingber, D. E. \& Mazur, E. (2005). Ablation of cytoskeletal filaments and mitochondria in live cells using a femtosecond laser nanoscissor, Mechanics and Chemistry of Biosystems 2, 17-26

Sidhu, M. S.; Kim, E. K; Woo, S. Y; Song. M. C.; Jeoung, S. C. \& Park, Y. I. (2009) Femtosecond - laser - assisted optoperforation of the primary retinal blood vessel and retina tissue of porcine eyes, J. Kor. Phys. Soc. 55(2) (in Press)

Singer, S. J. \& Nicolson, G. L. (1972). The fluid mosaic model of the structure of cell membranes, Science 175, 720-731

Strubinska, J. \& Sniezko, R. (2000). Localization of vegetative nucleus and generative cell nuclei in branching pollen tubes of Oenothera hookeri L. grown in vitro, Acta Biologica Cracoviensia Series Botanica 42, 107-112

Supatto, W.; Debarre, D.; Moulia, B.; Brouzes, E.; Martin, J. L.; Farge, E. \& Beaurepaire, E. (2005). In vivo modulation of morphogenetic movements in Drosophila embryos with femtosecond laser pulses, Proc. Natl. Acad. Sci. USA 102, 1047-1052

Tang, W.; Weidner, D. A.; Hu, B. Y.; Newton, R. J. \& Hu, X. H. (2006). Efficient delivery of small interfering RNA to plant cells by a nanosecond pulsed laser-induced stress wave for posttranscriptional gene silencing, Plant Science 171, 375-381

Taylor, L. P. \& Hepler, P. K. (1997). Pollen germination and tube growth, Anuu. Rev. Plant Physiol. Plant Mol. Biol. 48, 461-491

Tirlapur, U. K. \& König, K. (2002). Targeted transfection by femtosecond laser, Nature 418, 290-291

Touraev, A.; Stoger, E.; Voronin, V. \& Heberle-Bors, E. (1997). Plant male germ line transformation, Plant Journal 12, 949-956

Van der Leede-Plegt, L. M.; van den Ven, B. C. E.; Schilder, M.; Franker, J. \& van Tunen, A. J. (1995). Development of a pollen-mediated transformation method for Nicotiana glutinosa, Transgenic Res. 4, 77-86

Velegol, S. B.; Pardi, S.; Li, X.; Velegol, D. \& Logan, B. E. (2003). AFM imaging artifacts due to bacterial cell height and AFM tip geometry, Langmuir 19, 851-857

Vervaeke, I.; Londers, E.; Piot, G.; Deroose, R. \& Deproft, M. P. (2005). The division of the generative nucleus and the formation of callose plugs in pollen tubes of Aechmea fasciata (Bromeliaceae) cultured in vitro. Sexual plant reproduction 18, 9-19

Vogel, A. \& Venugopalan, V. (2003) Mechanism of pulsed laser ablation of biological tissues, Chem. Rev. 103, 577-644

Vogel, A.; Noack, J.; Huttman, G. \& Paltauf, G. (2005) Mechanism of femtosecond laser nanosurgery of cells and tissues, Appl. Phys. B 81, 1015-1047

Wang, Y.; Botvinick, E. L.; Zhao, Y.; Berns, M. W.; Usami, S.; Tsien, R. Y. \& Chien, S. (2005). Visualizing the mechanical activation of Src, Nature 434, 1040-1045

Yahng, J. S.; Jeoung, S. C.; Choi, D. S.; Cho, D.; Kim, J. H.; Choi, H. M. \& Paik, J. S. (2005). Fabrication of microfluidic devices by using a femtosecond laser micromachining techniques and $\mu$-PIV studies on its fluid dynamics, J. Korean Phys. Soc. 47, 977-981

Yamazaki, D.; Kurisu, S. \& Takenawa, T. (2005). Regulation of cancer cell motility through actin reorganization, Cancer. Sci. 96, 379-386

Zeira, E.; Manevitch, A.; Khatchatouriants, A.; Pappo, O.; Hyam, E.; Darash-Yahana, M.; Tavor, E.; Honigman, A.; Lewis, A. \& Galun, E. (2003). Femtosecond infrared laser An efficient and safe in vivo gene delivery system for prolonged expression, Molecular Therapy 8, 342-350 


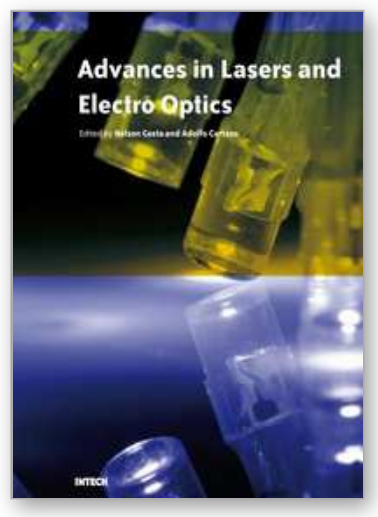

\author{
Advances in Lasers and Electro Optics \\ Edited by Nelson Costa and Adolfo Cartaxo
}

ISBN 978-953-307-088-9

Hard cover, 838 pages

Publisher InTech

Published online 01, April, 2010

Published in print edition April, 2010

Lasers and electro-optics is a field of research leading to constant breakthroughs. Indeed, tremendous advances have occurred in optical components and systems since the invention of laser in the late $50 \mathrm{~s}$, with applications in almost every imaginable field of science including control, astronomy, medicine, communications, measurements, etc. If we focus on lasers, for example, we find applications in quite different areas. We find lasers, for instance, in industry, emitting power level of several tens of kilowatts for welding and cutting; in medical applications, emitting power levels from few milliwatt to tens of Watt for various types of surgeries; and in optical fibre telecommunication systems, emitting power levels of the order of one milliwatt. This book is divided in four sections. The book presents several physical effects and properties of materials used in lasers and electro-optics in the first chapter and, in the three remaining chapters, applications of lasers and electro-optics in three different areas are presented.

\title{
How to reference
}

In order to correctly reference this scholarly work, feel free to copy and paste the following:

Sae Chae Jeoung, Mehra Singh Sidhu, Ji Sang Yahng, Hyun Joo Shin and GuYoun Baik (2010). Application of Ultrafast Laser Optoperforation for Plant Pollen Walls and Endothelial Cell Membranes, Advances in Lasers and Electro Optics, Nelson Costa and Adolfo Cartaxo (Ed.), ISBN: 978-953-307-088-9, InTech, Available from: http://www.intechopen.com/books/advances-in-lasers-and-electro-optics/application-of-ultrafast-laseroptoperforation-for-plant-pollen-walls-and-endothelial-cell-membranes

\section{INTECH}

open science | open minds

\section{InTech Europe}

University Campus STeP Ri

Slavka Krautzeka 83/A

51000 Rijeka, Croatia

Phone: +385 (51) 770447

Fax: +385 (51) 686166

www.intechopen.com

\section{InTech China}

Unit 405, Office Block, Hotel Equatorial Shanghai

No.65, Yan An Road (West), Shanghai, 200040, China

中国上海市延安西路65号上海国际贵都大饭店办公楼405单元

Phone: +86-21-62489820

Fax: $+86-21-62489821$ 
(C) 2010 The Author(s). Licensee IntechOpen. This chapter is distributed under the terms of the Creative Commons Attribution-NonCommercialShareAlike-3.0 License, which permits use, distribution and reproduction for non-commercial purposes, provided the original is properly cited and derivative works building on this content are distributed under the same license. 